\title{
Influence of Blade Aerodynamic Model on Prediction of Helicopter Rotor High-Frequency Airloads
}

\author{
Mary E. Kelly* \\ University of Glasgow, Glasgow, Scotland G12 8QQ, United Kingdom \\ and \\ Richard E. Brown \\ University of Strathclyde, Glasgow, Scotland G1 1XJ, United Kingdom \\ DOI: $10.2514 / 1 . C 031086$
}

\begin{abstract}
Brown's vorticity transport model has been used to investigate the influence of the blade aerodynamic model on the accuracy with which the high-frequency airloads associated with helicopter blade-vortex interactions can be predicted. The model yields an accurate representation of the wake structure yet allows significant flexibility in the way that the blade loading can be represented. A simple lifting-line model and a somewhat more sophisticated liftingchord model, based on unsteady thin aerofoil theory, are compared. A marked improvement in the accuracy of the predicted high-frequency airloads of the higher harmonic control aeroacoustic rotor is obtained when the liftingchord model is used instead of the lifting-line approach, and the quality of the prediction is affected less by the computational resolution of the wake. The lifting-line model overpredicts the amplitude of the lift response to bladevortex interactions as the computational grid is refined, exposing the fundamental deficiencies in this approach when modeling the aerodynamic response of the blade to interactions with vortices that are much smaller than its chord. The airloads that are predicted using the lifting-chord model are relatively insensitive to the resolution of the computation, and there are fundamental reasons to believe that properly converged numerical solutions may be attainable using this approach.
\end{abstract}

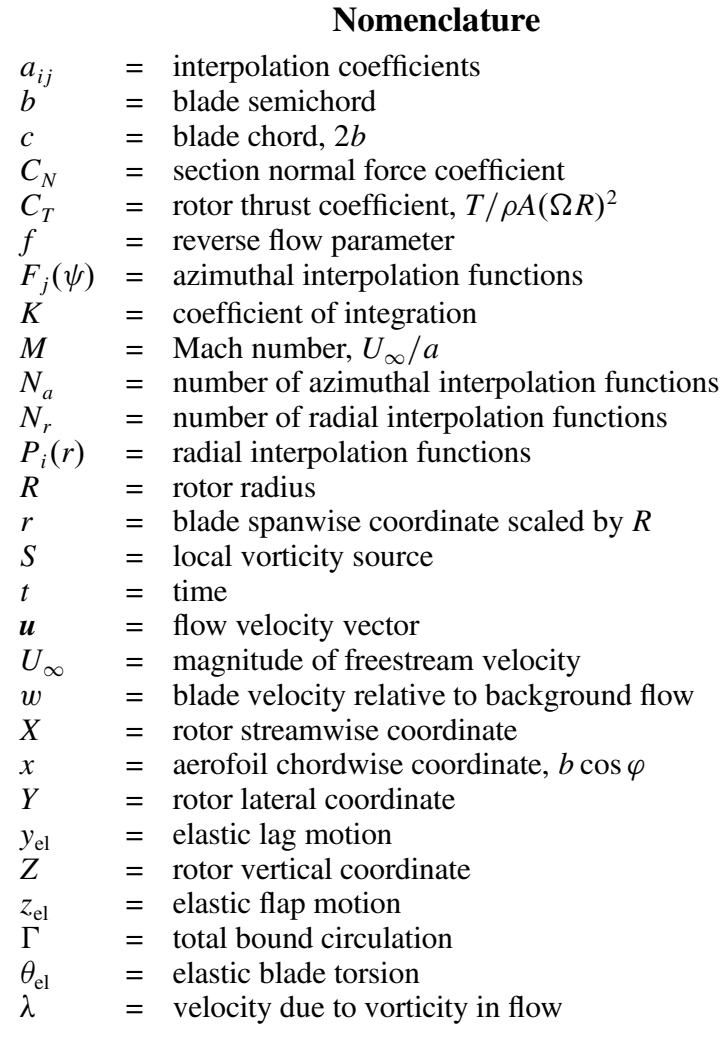

Received 27 April 2010; revision received 26 October 2010; accepted for publication 29 October 2010. Copyright (C) 2010 by Mary E. Kelly and Richard E. Brown. Published by the American Institute of Aeronautics and Astronautics, Inc., with permission. Copies of this paper may be made for personal or internal use, on condition that the copier pay the $\$ 10.00$ per-copy fee to the Copyright Clearance Center, Inc., 222 Rosewood Drive, Danvers, MA 01923; include the code 0021-8669/11 and \$10.00 in correspondence with the CCC.

*Department of Aerospace Engineering.

'Department of Mechanical Engineering.

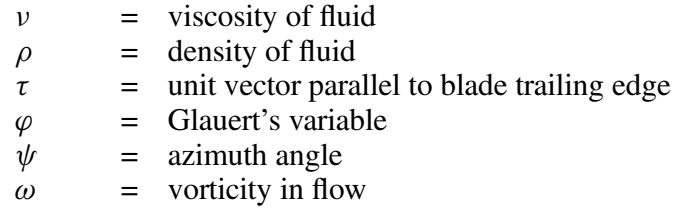

\section{Introduction}

A PARTICULAR source of helicopter vibration and noise, especially in descending flight or during maneuvers, is the localized aerodynamic interaction between the rotor blades and the vortices that they produce in their wake. Accurate prediction of the amplitude and position of the loading perturbations on the rotor that are induced by these blade-vortex interactions (BVI) relies critically upon the correct simulation of both the blade deformation and the position and strength of the vortical structures within the rotor wake. Modeling the evolution of the vortices within the wake, and correctly capturing their position and strength over time, is a particularly challenging task given the complex aerodynamic environment in which the helicopter rotor operates. The availability of a reliable tool that can accurately predict the high-frequency components of the blade loading, particularly those that are responsible for the rather objectionable characteristics of the helicopter under certain flight conditions, would be of significant benefit to the designers of modern rotorcraft given the current industrial focus on reduction of both maintenance costs and noise. As a result, accurate modeling of the structure of the wake generated by the helicopter rotor has become one of the primary challenges for the developers of computational fluid dynamics (CFD) methods designed for rotorcraft applications.

CFD calculations of the flow around the entire rotorcraft, or even just the rotor, are extremely challenging. This is because simultaneous accurate representation of flow features on the scale of the rotor (for instance, the overall geometry of the wake) and of vortex cores (for instance, the details of the roll-up process behind the blades that leads to the formation of the tip vortices) requires a method that can resolve the relevant physics over at least two spatial orders of magnitude. Nevertheless, recent advances in coupling rotorcraft computational structural dynamic (CSD) analyses to rotorcraft CFD 
have demonstrated significant progress in accurately predicting the rotor blade motion and capturing the associated blade airloads. Boyd [1] and Lim and Strawn [2], for instance, show encouraging results in comparison with the HĀRT II experimental data for prediction of BVI airloads and acoustics. Their investigations show, however, that high grid densities (with cell numbers of up to 113 million) are required to reduce the numerical dissipation of standard CFD techniques to a level where the high-frequency character of the BVIgenerated airloads can be captured. For most CFD methods, however, the accuracy of the solution is, inevitably, a compromise between the need for high-fidelity resolution of the wake and the computational cost that is incurred in achieving this fidelity. At present, this invariably results in solutions that are grid-dependent, given the prohibitive computational cost of rotor calculations on grids that are sufficiently fine to resolve the detailed structure of the wake.

The higher harmonic control aeroacoustics rotor test (HART) program [3-6] was initiated to provide experimental insight into the structure of the rotor wake and its effect on the aerodynamic loading of the rotor blades, and thus on the acoustic signature of the rotor. Three different cases were studied: a baseline (BL) case with conventional control inputs and two cases with higher harmonic control (HHC) inputs applied to the rotor (the so-called MV and MN cases). The second HART test, in particular, concentrated on gathering detailed measurements of the rotor wake in order to catalog the development of the blade-tip vortex as it ages and convects away from its point of origin. The three-component particle-imagevelocimetry (3C-PIV) technique that was used in the HART II tests allowed instantaneous measurement of the velocity field contained within a series of highly resolved observation areas.

There is much interest, especially in the context of rotor design, in developing methods that offer high-fidelity resolution of the rotor wake but at reduced computational cost compared with full CFD calculations. Lifting-line-type or lifting-surface-type aerodynamic models have been used widely in so-called comprehensive codes to provide blade airload information [7-9]. These models are generally inviscid in nature, and so the wake produced by the blades is modeled separately in terms of the trailed and shed vorticity from the blades. In many cases, it is only the trailed vortices that are modeled, and the shed vorticity is accounted for separately by an additional unsteady aerodynamic model that is then forced to rely upon a certain degree of empiricism. Additional considerations are required to account for compressibility effects and stall. Such models are relatively simple and easy to implement, but the physical accuracy of their portrayal of the dynamics of the wake, especially if based on a prescribed- or freewake approach, can be called into question, especially in terms of resolving the detail of the close blade-wake encounters associated with BVI. It is not clear if discrepancies in the wake model (or, instead, in the blade aerodynamic model that lies at the source of the wake) are responsible for the deficiencies in prediction of the BVIinduced loads that appear to be characteristic of this type of approach.

In this paper, the aerodynamics of the HART II rotor are modeled using Brown's vorticity transport model (VTM) [10,11], and results are compared with the HART II experimental data set [3-6]. The VTM is based on a time-dependent vorticity-velocity formulation of the Navier-Stokes equations, solved computationally on a structured grid system surrounding the rotor. The method has been designed specifically to reduce numerical dissipation of the vorticity in the flow, thus maintaining the integrity over many rotor revolutions, of the vortical structures that are present in the rotor wake (for instance, see Fig. 1). This paper aims to confirm that the VTM is well suited to modeling the geometry of the wake to the accuracy and detail that is required for the high-frequency components of the blade airload that are associated with BVIs to be captured correctly. To decouple the structural dynamic problem from the aerodynamic one, the structural deflection of the rotor blades was prescribed within the computational model. The prescription of the blade dynamics was based on a variable-separable interpolation of the blade deformations, which were measured during the HART II experiments [3-6].

The VTM allows significant flexibility in the way that the source of vorticity into the wake can be generated. For instance, a simple

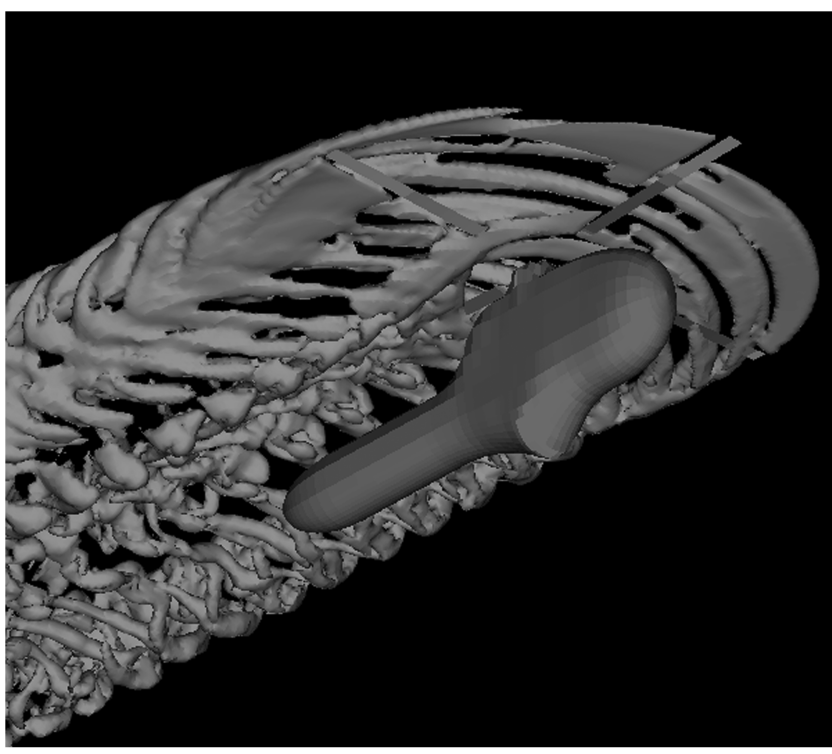

Fig. 1 VTM-calculated wake structure of the HART II system, visualized as a surface within the flow on which the vorticity has uniform magnitude.

lifting-line model for blade aerodynamics can be used, or a full, primitive-variable CFD calculation of the blade flow can be embedded in the calculation [12]. This flexibility makes the VTM ideal for studying the effect of the blade aerodynamic model on the fidelity of the prediction of the high-frequency BVI-induced loads on the rotor. The VTM framework has been used to predict the geometry of the wake system and the resultant rotor blade loading for the HART II rotor, using two different models for the blade aerodynamics.

The first blade aerodynamic model is relatively simple and is based on an extension of the Weissinger-L formulation of lifting-line theory. In this model, the strength of a bound vortex attached to the quarter chord of the blade is determined by enforcing a zero throughflow boundary condition at one discrete point located at the three-quarter chord of the airfoil. Results obtained using this approach are contrasted with the results obtained using a more sophisticated blade aerodynamics model. This model is based on an extension of classical unsteady thin-airfoil theory and uses Peters et al.'s state-space formulation for the blade airloads [13]. In this socalled lifting-chord approach, the aerodynamic environment of the blade is represented via a series of integrals over the chord of the blade, thus yielding a higher-order approximation to the aerodynamic loading on the blade than is given by lifting-line theory.

\section{Computational Model}

The present formulation of the VTM, developed by Brown and Line [10] and Brown [11] couples a model for the aerodynamics of the blades of the helicopter rotor to an Eulerian representation of the dynamics of the vorticity in the flowfield surrounding the helicopter.

\section{A. Wake Model}

The vorticity in the flowfield is evolved by the solution of the Navier-Stokes equations in vorticity-velocity form on a structured Cartesian grid surrounding the rotor. Assuming incompressible flow with velocity $\mathbf{u}$, the associated vorticity distribution $\omega=\nabla \times \mathbf{u}$ evolves according to the unsteady vorticity transport equation

$$
\frac{\partial}{\partial t} \omega+\mathbf{u} \cdot \nabla \omega-\omega \cdot \nabla \mathbf{u}=S+v \nabla^{2} \omega
$$

In this formulation, the vorticity then becomes the conserved variable within the flow and is thus not affected by the numerical dissipation that is inherent in CFD codes based on a pressure-velocity formulation of the Navier-Stokes equations. Toro's weighted average flux 
method [14] is used within the code to convect the solution through time. Within the particular implementation that is used, the local rate of numerical diffusion of vorticity is controlled very effectively by using a set of highly compressive flux limiters.

At each time step, the velocity at the cell faces is obtained from the vorticity distribution using a fast multipole technique to invert the differential form of the Biot-Savart equation

$$
\nabla^{2} \mathbf{u}=-\nabla \times \omega
$$

A semi-Lagrangian adaptive grid is used to track the evolving vorticity in such a way that cells only exist in regions of the computational domain where the vorticity is nonzero. As the vorticity moves to a new location, new cells are created, and any cells that no longer contain vorticity are destroyed. Thus, the grid structure is free to follow the evolution of the wake, eliminating the requirement for explicit numerical boundary conditions at the edge of the computational domain and increasing the computational efficiency of the method. Moreover, a nested grid system allows for fine resolution close to the rotor and then a systematic decrease in resolution with distance from the rotor hub.

\section{B. Blade Aerodynamic Model}

Two separate blade models have been incorporated within this VTM framework in order to yield the aerodynamic loading on the blades. In the first model, an extension of the Weissinger-L formulation of the lifting-line theory is implemented on a series of discrete panels along the length of each rotor blade. A bound vortex is attached to the quarter chord of each panel. The strength of the bound circulation $\Gamma$ along the length of the blade is then determined by enforcing, simultaneously, a condition of zero throughflow at the set of collocation points that are located at the three-quarter chord of each panel.

A second model for the blade aerodynamics is based on an extension of the classical unsteady thin aerofoil theory and uses a particular formulation for the airloads that is based on that developed in state-space form for flexible aerofoils by Peters et al. [13]. The zero throughflow boundary condition allows the total bound circulation on the aerofoil to be written as

$$
\Gamma=2 \pi b\left[f\left(w_{0}-\lambda_{0}\right)+\frac{1}{2}\left(w_{1}-\lambda_{1}\right)\right]
$$

where $f$ is a reverse flow parameter designed to enforce the Kutta condition at the downwind edge of the aerofoil. The circulation is defined in terms of the weighted integrals, given by

$$
\lambda_{n}=\frac{1}{K_{n}} \int_{0}^{\pi} \lambda \cos ^{n} \varphi \mathrm{d} \varphi
$$

and

$$
w_{n}=\frac{1}{K_{n}} \int_{0}^{\pi} w \cos ^{n} \varphi \mathrm{d} \varphi
$$

where $w$ is the component, normal to the blade chord, of the blade velocity relative to the uniform background flow, and $\lambda$ is the component, again normal to the blade chord, of the velocity due to all vorticity in the computational domain, except that which is bound to the panel under consideration. Glauert's variable $\varphi$ is defined such that

$$
x=b \cos \varphi, \quad-b \leq x \leq+b, \quad 0 \leq \varphi \leq \pi
$$

and

$$
K_{n}=\int_{0}^{\pi} \cos ^{n} \varphi \mathrm{d} \varphi
$$

These integrals are evaluated numerically after evaluating the integrands at several discrete points along the chord of each blade panel. The sectional lift (per unit span) is then given by [13] as

$$
L=\rho U_{\infty}\left(\Gamma+\frac{1}{2} \lambda_{1}\right)+\pi \rho b^{2} \dot{w}_{0}
$$

Using the same notation, the total bound circulation on the aerofoil given by the Weissinger- $\mathrm{L}$ method is

$$
\Gamma=\left.2 \pi b f[w-\lambda]\right|_{x=b / 2}
$$

and the sectional lift (per unit span) is simply

$$
L=\rho U_{\infty} \Gamma+\left.\pi \rho b^{2} \dot{w}\right|_{x=0}
$$

In both aerodynamic models, the trailed and shed vorticities from each vortex panel are added to the near wake downstream of the blade as the local vorticity source:

$$
S=-\tau \frac{\partial \Gamma}{\partial t}+\mathbf{u}_{b} \frac{\partial \Gamma}{\partial r}
$$

where $\tau$ is the unit vector parallel to the trailing edge of the blade, and $\mathbf{u}_{b}$ is the velocity of the trailing edge relative to the air.

The two-dimensional aerodynamic characteristics of the rotor blade sections are specified in a lookup table as a function of the angle of attack and Mach number for a given Reynolds number. These characteristics can be used to precondition the zero throughflow boundary condition to allow the blade aerodynamic calculation to closely match the sectional aerodynamic characteristics, including stall, of the actual blade. As this approach is still essentially inviscid, the profile drag of the blade is calculated as a separate function of local angle of attack and is then added to the local aerodynamic force that is calculated from the blade aerodynamic model.

\section{Fuselage Model}

Fuselages or other solid bodies are represented using an unsteady vortex panel method, as described in [15]. The surface of any body immersed in the flowfield is discretized into a system of panels, such that each panel edge is represented as a vortex filament with constant strength, forming a closed loop of vorticity. The velocity at the centroid of any panel is calculated as the sum of the influences from all vortex filaments on the body together with the velocity induced by all the other vorticity within the flow. To determine the strengths of the vortex loops, a boundary condition of zero throughflow is enforced simultaneously at the centroids of all panels. Where present in the simulations described in this paper, the drive housing for the HART II rotor was modeled using 1908 panels. This yields a level of resolution that is comparable to previous simulations using this approach (for example, as described in [15]).

\section{Structural Dynamics}

In the particular version of the model used in the present investigation, the motion of the blades is prescribed based on a variableseparable interpolation of the blade deformations that were measured at discrete azimuthal and radial locations on each blade during the HART II experiment. In the experiment, the blade deformation was measured using a nonintrusive optical method called stereo pattern recognition, as described in [16-18].

Each component $D$ of the blade deformation is reconstructed in the simulations by using interpolating functions of the form

$$
D(r, \psi)=\sum_{i=1}^{N_{r}} \sum_{j=1}^{N_{a}} a_{i j} P_{i}(r) F_{j}(\psi)
$$

where $N_{r}$ and $N_{a}$ are, respectively, the number of radial and azimuthal interpolation functions, $P_{i}(r)$ and $F_{j}(\psi)$, used to describe the particular component of the blade deflection. The radial interpolation functions were taken to be polynomials, and the azimuthal interpolation functions were taken to be the components of a Fourier series, so that

$$
P_{i}(r)=r^{(i-1)}
$$


and

$$
F_{j}(\psi)= \begin{cases}\cos \frac{j-1}{2} \psi, & \text { if } j \in\{1,3,5, \ldots\} \\ \sin \frac{j}{2} \psi, & \text { if } j \in\{2,4,6, \ldots\}\end{cases}
$$

The coefficients $a_{i j}$ of the interpolation function were calculated by enforcing a simple least-squares fit to the measured data for the blade deformations. This method interpolates over the relatively sparse experimental data, as well as fills any gaps in the data where the markers used in the measurements could not be viewed because they laid within the shadow of the drive enclosure and the mounting support or had peeled off the blades. The sets of coefficients that give the best approximation to the elastic flap, lag, and torsional deformations $\left(z_{\mathrm{el}}, y_{\mathrm{el}}\right.$, and $\left.\theta_{\mathrm{el}}\right)$ of the blades using six radial and nine azimuthal interpolation functions are given, at least for the HART II BL case, in the appendix to [19].

The difference between the interpolation and the experimental data set for each of the measured components of the elastic deformation of the blades was within the stated error bounds on the measurements of $\pm 0.5^{\circ}$ for the elastic torsion and $\pm 0.5 \mathrm{~mm}$ for the flap and lag deflections. Nevertheless, the reliability of the interpolation may be questioned in areas where the experimental data were particularly sparse, as was, for instance, the case around 0 and $180^{\circ}$ azimuth

\section{Airload Predictions}

The aerodynamic environment that is encountered by the rotor blades of a helicopter under typical operating conditions is highly unsteady. Although the main contributions to the blade angle of attack come from the control inputs and the elastic deformations, additional and not insignificant effects are introduced by the wake that is produced by the rotor in flight. In particular, the concentrated vortices that are trailed from the tips of the blades induce rapid changes in angle of attack over very short time scales when they encounter a blade. To capture the effects of these interactions on the loading, the blade aerodynamic model must be able to capture the aerodynamic response of the blade when subjected to these very transitory perturbations to its aerodynamic environment. Rapid transients in both the angle of attack of each blade section and the rate of change of the angle of attack are caused by the pitching motions of the blade. In addition, the flapping motion of the blade results in a plunging motion at each blade section, and changes in the freestream flow cause each blade section to experience a nonuniform gust field.

The unsteady aerodynamic response of the blade sections to each of these stimuli is different; thus, the combined response can be extremely complex [20]. It is important that the model that is used to represent the aerodynamics of the blade is able to distinguish between the effects on the blade airload that arise from each of the stimuli mentioned previously. In particular, the model that is used must be able to correctly represent both the unsteady response of the blade due to changes in angle of attack and the response that is due to a vertical gust velocity normal to the blade. The ability of each of the two blade aerodynamic models to distinguish between these two phenomena can be ascertained by comparing their predicted response to the analytical solutions to Wagner's [21] and Küssner's [22] classical problems from the field of linearized, unsteady aerofoil aerodynamics. As a prerequisite to the accurate prediction of BVI noise, it is also important to verify the ability of each of the blade models to accurately predict the aerodynamic response of an aerofoil to an encounter with an isolated vortex. The characteristics of the two blade models in this respect are investigated in the following sections of this paper.

\section{A. Wagner's Problem}

Wagner's problem [21] considers the transient lift response of a thin aerofoil of chord $2 \bar{b}$ to a step change in angle of attack (from zero to $\alpha$ at time $t=0)$. Wagner showed that the lift response due to circulation was of the form

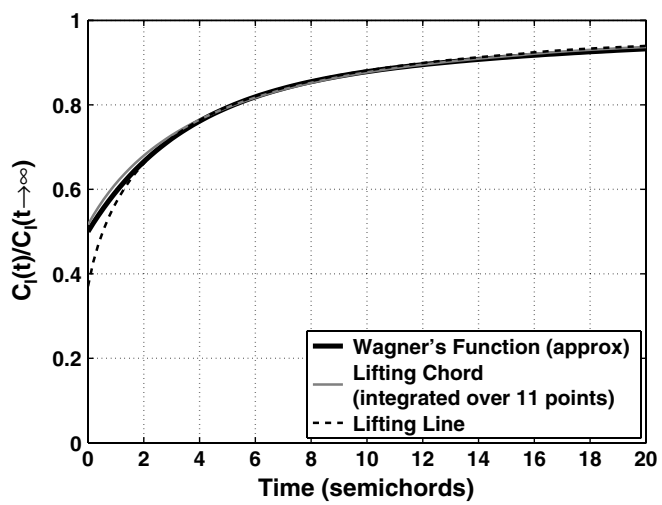

Fig. 2 Wagner's problem [21] for the aerodynamic response of an aerofoil to a step change in angle of attack.

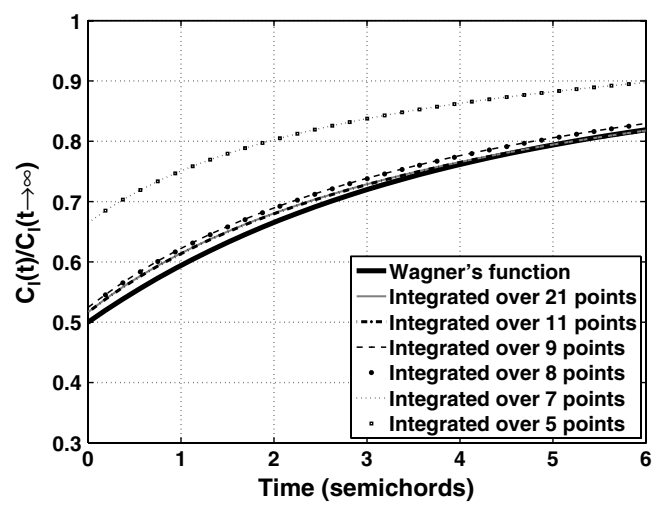

Fig. 3 Convergence of the lifting-chord model to Wagner's exact solution [21] as the number of points is increased over which the liftingchord integrals are evaluated.

$$
\frac{C_{l}(t)}{C_{l}(t \rightarrow \infty)}=\phi(s)
$$

assuming that $\alpha$ is small, where $\phi(s)$ is known as Wagner's function and $s=U_{\infty} t / b$ : i.e., the number of semichords traveled by the aerofoil in time $t$. Wagner's function has an initial value of one-half and asymptotes to unity as time goes to infinity.

The lift response of an aerofoil,,$\stackrel{ \pm}{ \pm}$ as predicted using the lifting-line model and the lifting-chord model, to a step change in angle of attack is compared with Wagner's analytical result in Fig. 2 [21]. This figure shows the reasonably well-known fact that the initial lift predicted by the lifting-line model is equal to one-third of the steady-state lift rather than one-half of the steady-state value, whereas the liftingchord approach reproduces Wagner's exact solution more accurately.

Increasing the number of chordwise points over which the weighted integrals are evaluated increases the accuracy of the initial lift response of the lifting-chord model. Indeed, Fig. 3 shows the lifting-chord solution to effectively converge onto the analytical solution once the integrals are evaluated at 11 or more points along the aerofoil chord.

\section{B. Küssner's Problem}

In contrast to Wagner's problem [21], Küssner's problem [22] considers the response of an aerofoil to a transient change in angle of attack as the aerofoil enters and progresses through a sharp-edged vertical gust. The transient lift response can be written as

$$
\frac{C_{l}(t)}{C_{l}(t \rightarrow \infty)}=\psi(s)
$$

\footnotetext{
\# two-dimensional aerofoil is modeled in the VTM as a rotor blade with a very large aspect ratio.
} 


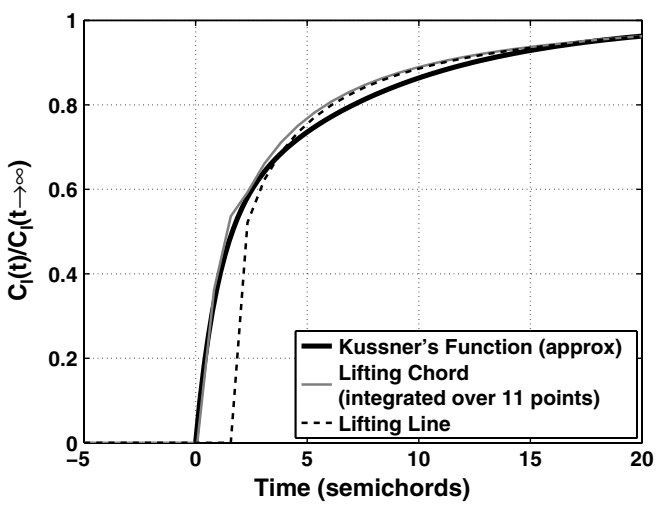

Fig. 4 Analytical and numerical solutions to Küssner's problem [22] compared. (The leading edge of the aerofoil encounters the gust at time $\boldsymbol{t}=\mathbf{0}$.)

where $\psi(s)$ is Küssner's function. Küssner's function has an initial value of zero and asymptotes to unity as time goes to infinity.

The aerodynamic response of an aerofoil to its progression through a sharp-edged gust, as predicted using the lifting-line model and the lifting-chord model, is compared with Küssner's analytical solution in Fig. 4 [22]. The lifting-chord approach captures Küssner's analytical result correctly, in the sense that the response to the gust starts as soon as the leading edge of the aerofoil penetrates the gust. In contrast, in the lifting-line case, the blade does not respond until the forward edge of the gust reaches the three-quarter chord of the aerofoil: in other words, the point at which the zero throughflow boundary condition is evaluated.

\section{Interaction with Isolated Vortex}

To illustrate the behavior of the two different blade models in capturing the BVI-induced aerodynamic response of the blade, Fig. 5 shows the predicted response of an aerofoil to an encounter with an isolated vortex. In the example shown in the figure, the vortex passes $0.25 c$ below the quarter-chord line of the aerofoil while convecting downstream at a Mach number of 0.626 . The core radius of the interacting vortex is 0.162 chords (i.e., $c / 6.172$ ), thus yielding a BVI of comparable dimensions to the simulated vortex core size encountered in the context of the numerical simulations of the HART II system described later in this paper. This is distinct from the physical core size of the interacting vortex, as measured in the HART II experiment, which is generally much smaller when compared with the chord of the blade.

This case allows the ability of each of the blade models to reproduce the highly impulsive airloads that are characteristic of BVIs to be examined in a more realistic context than that presented in the previous two sections of this paper. In Fig. 5, the predictions of the lifting-line and lifting-chord models are compared with the predictions of a conventional CFD method [23], which uses a

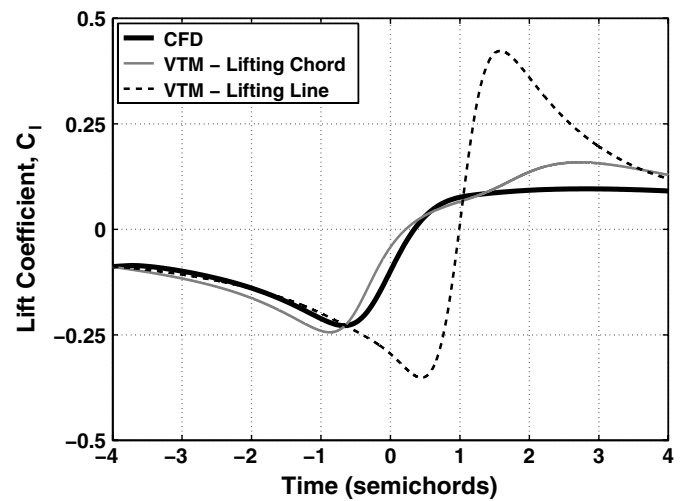

Fig. 5 Predicted aerodynamic response of an aerofoil to interaction with an isolated vortex. (The vortex passes $0.25 \mathrm{c}$ below the quarter chord of the aerofoil at time $t=0$.)

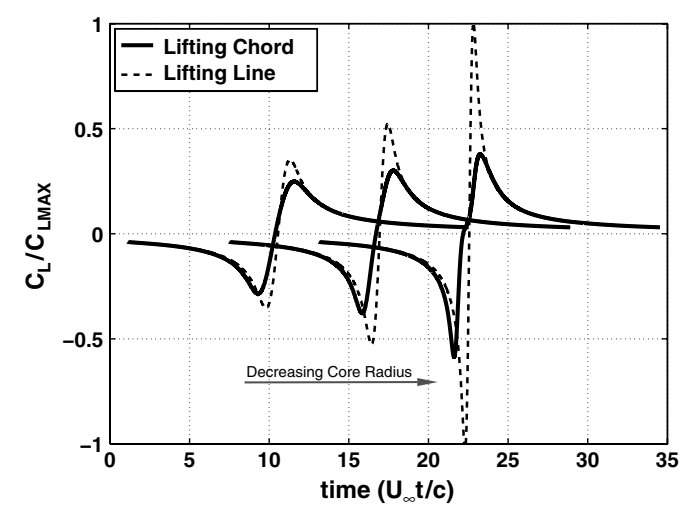

Fig. 6 Predicted aerodynamic response of an aerofoil to interaction with isolated vortices with varying core sizes but constant circulation (core size decreases from left to right).

pressure-velocity formulation of the Euler equations throughout the computational domain surrounding the aerofoil. This method is able to provide a more accurate representation of the pressure response of the aerofoil than the simpler lifting-line or lifting-chord models, and it compares favorably to experimental data for an interaction of these dimensions [24]. The lifting-line model responds much later to the vortex-induced flowfield than does the lifting-chord model and, in comparison, significantly overpredicts the maximum loading on the aerofoil that results from the interaction. Indeed, the loading on the aerofoil once the vortex has passed downstream of the aerofoil is far better predicted by the lifting-chord model than the lifting-line model, indicating a significantly better match between the circulation that is generated on the blade using this model and the vorticity that is shed into the wake downstream of the trailing edge of the aerofoil.

The relative sensitivity of the two blade models to the size of the interacting vortex is illustrated in Fig. 6, where the predicted response of an aerofoil to a direct interaction with a set of isolated vortices, each with the same circulation but with various core sizes, is shown. Both models predict an increase in the amplitude of the induced loading peak as the core size of the vortex is reduced but, using the lifting-line approach, the relative increase in amplitude is more significant. A slight phase shift is also visible between the predictions of the two blade models. The consequence of these observations for the accuracy of the prediction, not only of the BVIinduced airloads on the rotor but also of the subsequent structure of the wake, are explored later in this paper.

Each of the methods show fundamentally different behavior in the presence of BVI-scale features in the wake. Indeed, Figs. 5 and $\underline{6}$ suggest that the lifting-line approach, due to its single-point boundary condition, might misrepresent the vortex-induced airload on the blade, particularly when the interacting vortices have core sizes that are significantly smaller than the blade chord. In contrast, the lifting-chord model is much less sensitive to the detail contained within the velocity field and is, instead, reliant on the (weighted) integral of the velocity along the entire chord of the blade. The expected consequence is that this model might produce more robust predictions of the blade airloads that are reliant rather upon the invariant, integral properties of the vortex, such as its circulation. These results suggest that the quality of prediction of the highharmonic components of the blade airloads can be improved simply by paying careful attention to the fidelity of the model that is used to represent the aerodynamics of the blades.

\section{HART II Airload Prediction}

The airload as predicted by the VTM is compared with experimental data for the three cases of the HART II test: the BL case with conventional control inputs and the two cases with additional threeper-revolution HHC inputs applied to the rotor [the so-called minimum noise $(\mathrm{MN})$ and minimum vibration (MV) cases] [3]. The operational parameters of the HART II test are summarized in Table 1 . In addition, simulations at three different spatial and 
Table 1 Rotor operational parameters

\begin{tabular}{lc}
\hline \hline Parameter & Value \\
\hline Forward velocity & $33 \mathrm{~m} / \mathrm{s}$ \\
Rotational speed & $1041 \mathrm{rpm}$ \\
Blade passage frequency & $69.4 \mathrm{~Hz}$ \\
Shaft tilt & $5.3^{\circ}$ \\
Thrust coefficient & 0.00457 \\
Advance ratio & 0.151 \\
\hline \hline
\end{tabular}

Table 2 Computational resolution

\begin{tabular}{lcccc}
\hline \hline Size of smallest cells & $\begin{array}{c}\text { Time steps per } \\
\text { rotor revolution }\end{array}$ & $\begin{array}{c}\text { Degrees of azimuth } \\
\text { per time step }\end{array}$ \\
\hline Coarse & $R / 55.5$ & $c / 3.36$ & 350 & 1.03 \\
Medium & $R / 83.3$ & $c / 5.04$ & 525 & 0.69 \\
Fine & $R / 125.0$ & $c / 7.56$ & 800 & 0.45 \\
\hline \hline
\end{tabular}

temporal resolutions, as defined in Table 2, are compared to expose the effect of grid resolution on the ability of each of the blade models to predict the airloads on the rotor. Throughout, each blade was discretized into 40 spanwise panels. These panels were cosinedistributed along the span to improve resolution of the loading gradients near the root and tip of the blade, but no additional clustering was employed to improve resolution near the positions of individual BVI events. In all simulations, the rotor was trimmed to the experimental thrust coefficient and to zero aerodynamic pitch and zero roll moments about its hub. Throughout, the structural dynamics of the blades were prescribed using six interpolation functions in the radial direction and nine in the azimuthal direction.

Figure 7 shows the predicted normal force coefficient $C_{N} M^{2}$ for the HART II BL case, plotted as a series of contours over the rotor disk. The airload predicted by the VTM at each of the three different resolutions of the flowfield, defined in Table 2, and using the liftingline and lifting-chord models for the blades are compared. The BVIs are visible in the contour maps as thin ridges of relatively high loading toward the rear of the disk, in the first and fourth quadrants of the rotor. The interactions are strongest outboard toward the blade tips, particularly on the retreating side of the disk. Where the liftingline approach is used, an increase in grid resolution results in a marked increase in the amplitude of the BVI peaks, and the asso- ciated loading gradients become significantly steeper. In contrast, the lifting-chord approach shows much less sensitivity to the size of the computational cells.

Similar contours of the normal force coefficient for all three cases of the HART II test are shown in Fig. 8. In the BL case, the contours of highest loading occur in a relatively even band across the front of the disk. The loading in this band is higher in the MN case than in the BL case and is less even, with peaks in loading further outboard on the disk, toward the advancing and retreating sides. In the MV case, the peaks in the overall loading are shifted further forward on the retreating side and further aft on the advancing side of the disk when compared with the $\mathrm{MN}$ case. The changes in the location on the rotor disk where the BVI events occur with the addition of $\mathrm{HHC}$ are also apparent in these plots and are discussed in more detail later.

During the HART II test program, the sectional airload $C_{N}$ at $87 \%$ of the blade span was estimated by conditionally averaging the signal from a set of pressure transducers mounted at this section of the blade. The measured blade airload at the $87 \%$ radial station for the three HART II cases, expressed in terms of normal force coefficient $C_{N} M^{2}$, are compared in Fig. 9 to the loading predicted by the VTM. In general, the calculated loading at this radial station compares well with the experimental data for all cases. The advancing side is perhaps the least well resolved, with the largest discrepancies between prediction and experiment on this side of the disk occurring in the low-frequency component of the airload. The predictions of the loading on the retreating side of the disk correlate well with the experimental data for all three cases. The figures show, nevertheless, that the lifting-line method is more sensitive than the lifting-chord approach to changes in the cell size that are used to represent the wake.

\section{A. Low-Frequency Component of Airload}

Figure 10 shows the normal force coefficient $C_{N} M^{2}$ for all three HART II cases, after filtering at a frequency of 10 per rotor revolution to separate out the low-frequency component of the signal. These figures clearly illustrate that, in all three cases, the major differences between the experimental data and the numerically predicted lift for the low-frequency component of the signal lie mainly to the advancing side of the disk between 0 and $120^{\circ}$ azimuth. The discrepancies in this component of the airload are similar, regardless of computational resolution and irrespective of the model that is used to represent the aerodynamics of the blade.
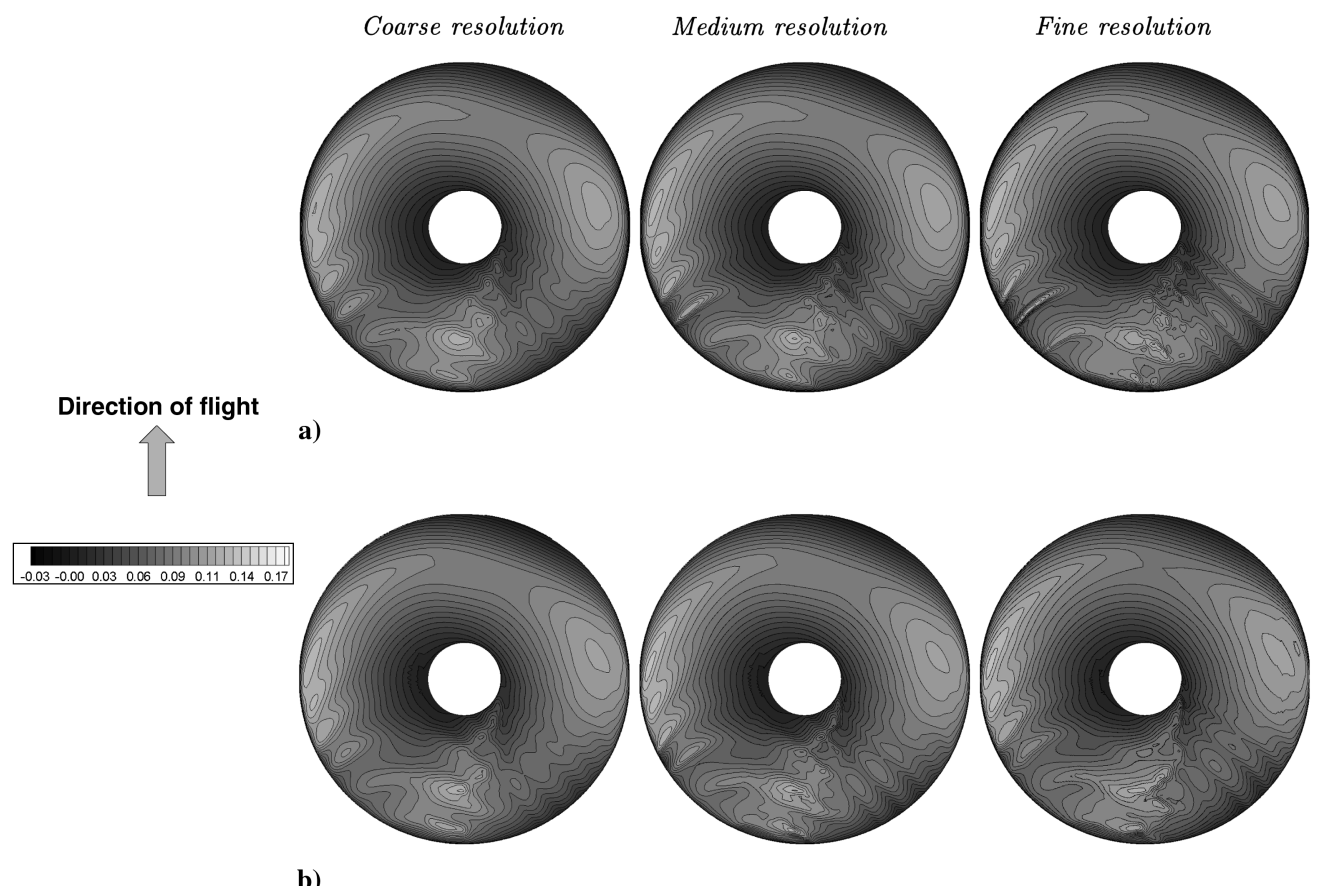

b)

Fig. 7 Contours of the normal force coefficient $C_{N} M^{2}$ as predicted by the VTM at various computational resolutions for the HART II BL case using the a) lifting-line and $b$ ) lifting-chord representations of the blade aerodynamics. 

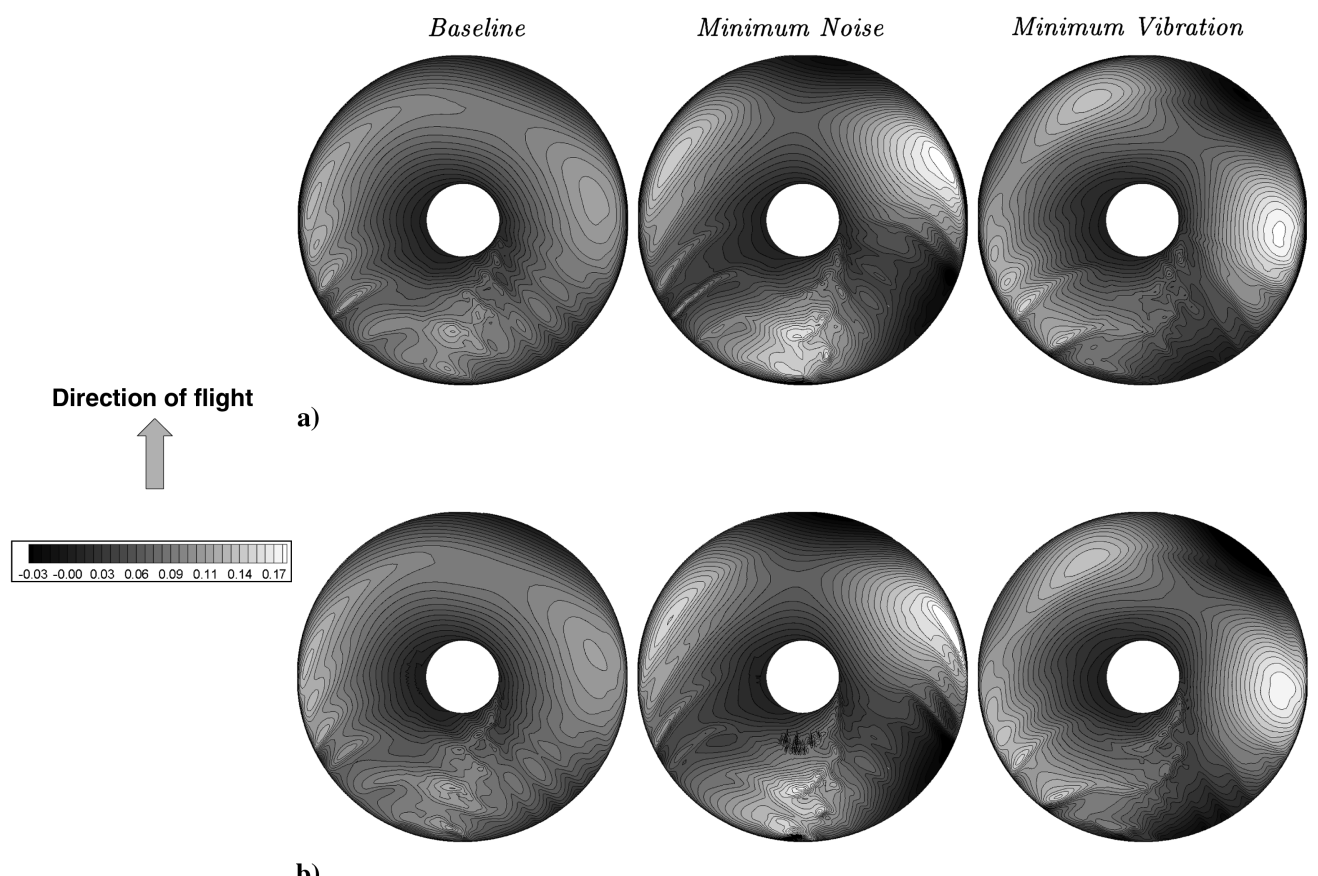

Fig. 8 Contours of the normal force coefficient $C_{N} M^{2}$ as predicted by the VTM at the medium computational resolution for all three HART II cases using the a) lifting-line and $b$ ) lifting-chord representations of the blade aerodynamics.

Several reasons for the observed discrepancies can be postulated. Incorrect prediction of the strength of the vorticity trailed from the roots of the blades might, for instance, adversely affect predictions of the flow and, hence, the loading experienced by the blades as they pass near the rear of the disk. A parametric study has shown, however, that there is very little qualitative improvement in the blade loading obtained by varying the boundary condition that is applied at the root of the blade, and hence to the strength of the vortices emanating from there.

An additional factor that should be considered is that the loading distribution on the advancing side of the HART II rotor under the experimental conditions is quite unusual in being almost uniformly spread along the length of the blade. This yields a sheet of trailed vorticity behind the blade that is relatively weak and thus takes some time to roll up to form a coherent tip vortex. This particular aspect of the vortical structure of the wake of the HART II rotor is shown later in the paper to be predicted very accurately by the VTM; hence, the contribution of any inaccuracy in prediction of this roll-up process to the observed errors in blade loading is, at most, very subtle.

As it is this component of the loading that is primarily affected by control inputs and blade structural deformation, it is most likely that the observed discrepancies in the prediction of the low-frequency component of the blade loading are simply due to errors in the interpolation that was used to prescribe the blade dynamics within the simulation. Indeed, it is suggestive that the most significant discrepancies between the experimental data for the torsional deformation of the blades and the interpolation used in the simulation coincide rather closely with these regions of the rotor disk. The missing experimental data for the structural deformation of the blades around 0 and $180^{\circ}$ azimuth may have had a particularly strong effect on the quality of the structural model on those regions of the advancing side of the rotor that are most strongly influenced by BVI. This explanation is supported by the calculations of Boyd [1] and Lim and Strawn [2], where a coupled CSD/CFD approach was used to substitute a numerical analysis of the structural dynamics of the rotor blades for the prescribed structural deflections used here. Their simulations yield particularly good correlations with experimental data for the low-frequency component of the blade airload.

\section{B. High-Frequency Component of Airload}

The high-frequency component of the blade airload is almost exclusively due to the localized interactions between the rotor blades and the vortices within the rotor wake. In Fig. 11, the contours of the predicted normal force coefficient $C_{N} M^{2}$ have been filtered to include only the high-frequency components of the signal (i.e., those greater than ten per rotor revolution) to expose in greater detail the distribution of these BVI events across the rotor disk.

Figure 11 illustrates the effect of HHC inputs on the position and strength of the BVIs. In the BL case, in which no HHC is applied, the BVI-loading peaks are located outboard on the rotor disk and toward its rear: i.e., in quadrants one and four. The interactions on the retreating side of the disk are stronger, as the interacting vortices are orientated more nearly parallel to the blade than on the advancing side (see Sec. V). In comparison with the BL case, the BVI events in the MN case, particularly within the first quadrant on the advancing side of the rotor, are located significantly more inboard. Since the Mach number of the blade increases toward the tip, those interactions that occur inboard take place at lower Mach numbers. Moreover, the tip vortices pass close to the disk further forward than in the BL case (again, see Sec. V); thus, the area of the disk over which the BVI events occur extends further forward. On the retreating side, again, the BVIs occur further forward in the MN case than in the BL case and, as a result, the interacting vortices are no longer orientated parallel to the blade. Both the reduction in Mach number where the interactions take place and the change in orientation of the interaction reduce the amount of noise that is radiated toward the ground.

In the MV case, the azimuthal locations of the BVI events remain similar to those of the BL case on both the advancing and retreating sides of the rotor, but the associated loading peaks are shifted more outboard toward the blade tips. The higher Mach numbers associated with these interactions produce an increase in the noise radiation from both sides of the disk. The trends observed in the VTM calculations are comparable to those obtained by plotting the experimentally measured pressure along the leading edge of the blade for the three HART II cases $[\underline{3}, \underline{4}]$. The computations also reveal the presence of relatively strong interactions with the root-vortex system that is produced by the blades. These interactions are not apparent in the experimental data, however, and may be an artifact of the omission of any representation of the rotor hub assembly from the calculations.

Figures 12-14 show the normal force coefficient $C_{N} M^{2}$ for the $87 \%$ radial station (at which pressure measurements were gathered during the HART II test, see Sec. IV) after it has been filtered to contain only the high-frequency component of the loading (i.e., that which is induced by the interactions between the blades and the 

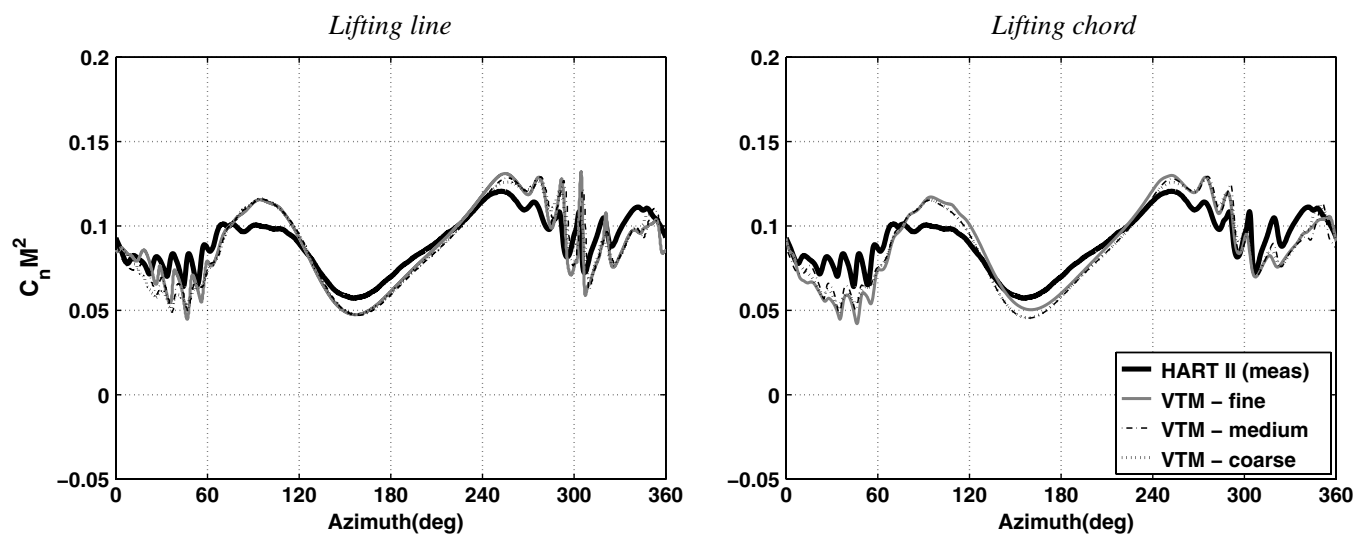

a) Baseline Case
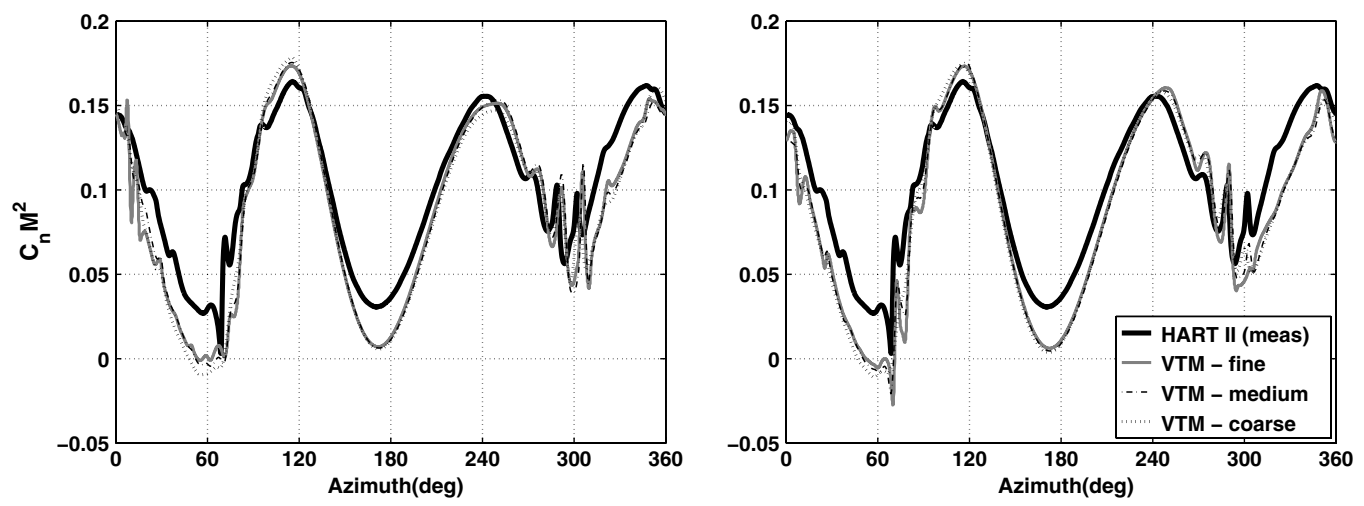

b) Minimum Noise Case
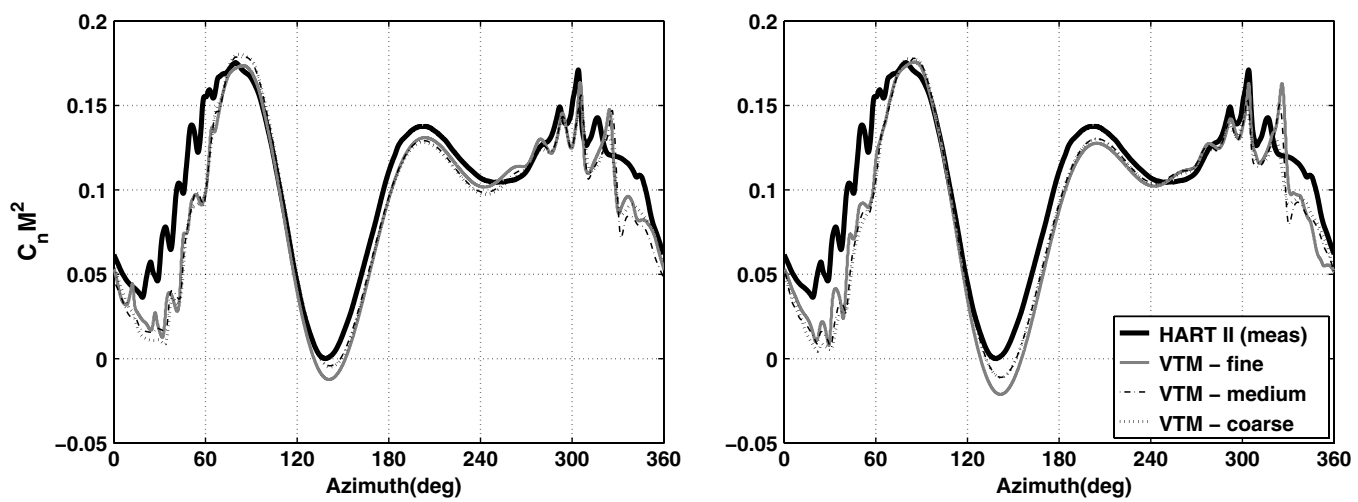

c) Minimum Vibration Case

Fig. 9 Comparison of normal force coefficient $C_{N} M^{2}$ at $87 \%$ span, as predicted using lifting-line and lifting-chord representations of the blade aerodynamics, against experimental data for all three HART II cases.

vortices in the wake). In these figures, the BVI-induced loading fields on the retreating and advancing sides of the rotor have been produced with expanded azimuthal scale to aid their interpretation.

For each of the HART II cases, the numerical representation of the BVI events on the retreating side of the rotor is generally satisfactory, apart from a rather gross misrepresentation of the amplitude and position of the furthest-aft BVI in the MV case. In addition, the predicted amplitude of this loading peak is very sensitive to the cell size used in the simulation. Indeed, the differences in the grid sensitivity of the two blade aerodynamic models in their prediction of the BVI-induced loading are most obvious on this side of the disk.

As the grid resolution is increased, the amplitudes of the peaks in the BVI-induced loading on the blade that are predicted by the liftingline model increase markedly, resulting in a gross overprediction in amplitude of the BVI-loading peaks at the finest computational resolution. As alluded to earlier, the deficiencies in prediction of the BVI-induced airloads when the lifting-line model is used are due to the misrepresentation of the aerodynamic response of the blade when subjected to the very localized perturbations in its aerodynamic environment that are characteristic of helicopter BVIs. This characteristic can be explained further in terms of the relative sensitivity of each of the blade aerodynamic models to the details of the velocity profile that is induced at the blade by the interacting vortex. As the grid is refined within the VTM, the vorticity that is associated with any particular vortex is confined to fewer cells. This results in a velocity profile for the vortex that becomes more spiky as the size of the computational cells that are used to resolve the flow are reduced. With its single-point boundary condition, the lifting-line model is overly sensitive to the maxima and minima within the velocity field that is encountered by the blade. In contrast, the lifting-chord model, forced as it is by the (weighted) integral of the velocity along the entire chord of the blade, is much less responsive to the detail contained within the velocity field and is instead more heavily influenced by the invariant, integral properties of the flowfield, such as its circulation or net vorticity. 

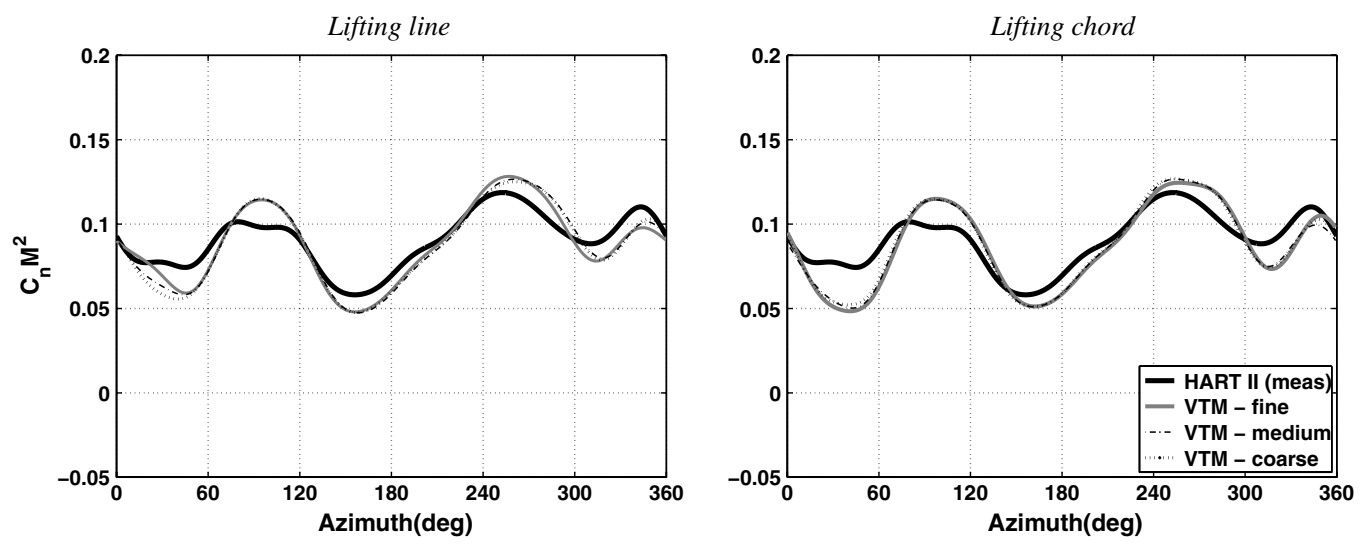

a) Baseline Case
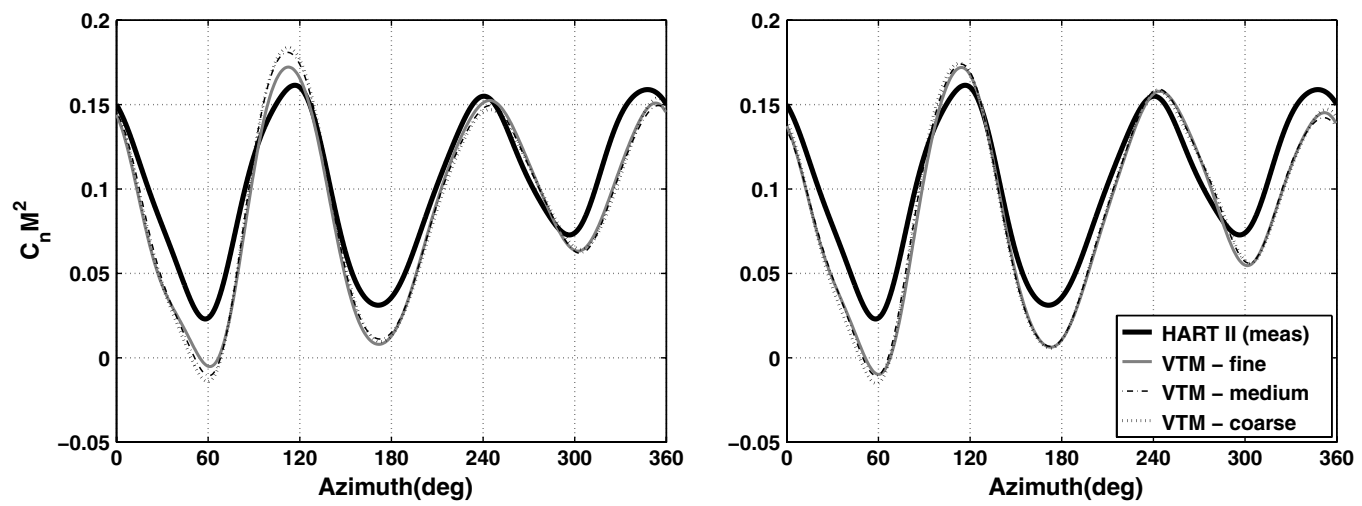

b) Minimum Noise Case
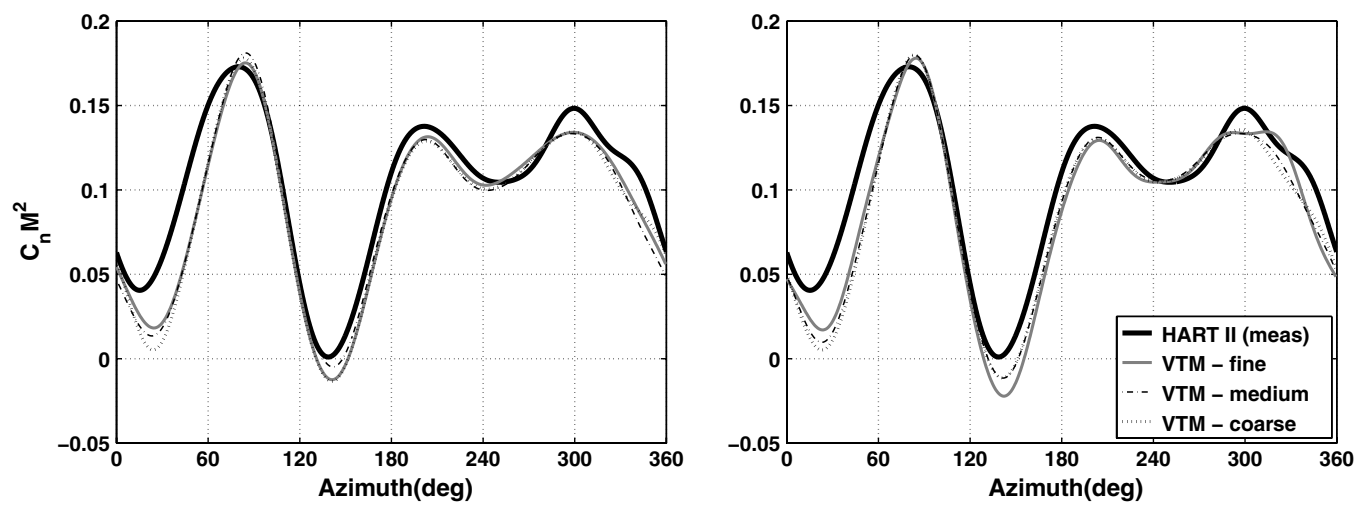

c) Minimum Vibration Case

Fig. 10 Comparison of low-frequency component of normal force coefficient $C_{N} M^{2}$ at $87 \%$ span, as predicted using lifting-line and lifting-chord representations of the blade aerodynamics, against experimental data for all three HART II cases.

In general, on the retreating side of the disk, a marked improvement in the accuracy of the predicted high-frequency airloads of the HART II rotor is obtained when the lifting-chord model for the blade aerodynamics is used instead of the lifting-line type approach.

The advancing side of the disk presents a somewhat more confusing picture, however. For all three HART II cases, all the BVIs on this side of the rotor are captured by the numerics, but there are errors in the phasing and amplitude of the BVI-induced loading peaks in almost all cases. The MN and MV cases (Figs. 13 and 14, respectively) show somewhat greater discrepancies between the predictions and the experimental data than the BL case. In the BL case, there is generally good agreement between measurement and prediction, but the strongest BVI event on the advancing side of the disk is slightly misrepresented in phase by the numerical method. In the measured signal, the loading peak with the largest amplitude occurs at approximately $50^{\circ}$ azimuth. In contrast, the numerical calculations using coarse- or medium-grid resolutions predict the BVI event that occurs at an azimuth of $40^{\circ}$ to be marginally stronger than the BVI at $50^{\circ}$. The predicted loading intensity is thus shifted toward the rear of the disk compared with experiment. At the finest computational resolution, however, the difference in magnitude between each of these two BVI impulses is minimal. It is believed that this sensitivity in the relative strength of these BVIs to the computational resolution has a strong influence on the predicted position of the acoustic maximum on the advancing side of the disk.

In the MN case, the most intense BVI impulse, at an azimuth of $70^{\circ}$ on the advancing side, is underpredicted in amplitude when the lifting-line model is used, regardless of the resolution of the flowfield. In contrast, as the grid resolution is increased the lifting-chord model very accurately captures this particular loading peak. This is discussed further in the next section of this paper. 

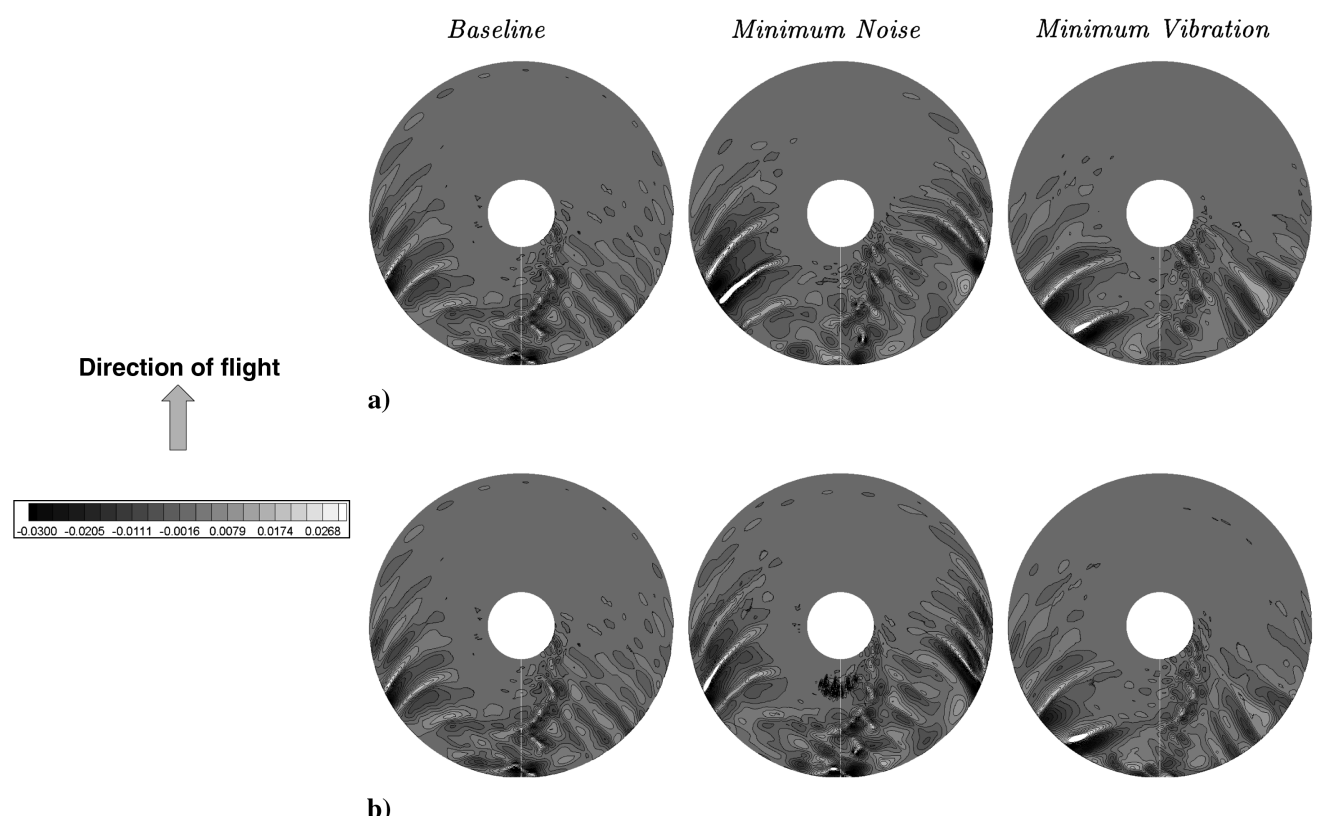

b)

Fig. 11 Contours of normal force coefficient $C_{N} M^{2}$, as predicted by the VTM at the medium computational resolution, using the a) lifting-line and b) lifting-chord representations of the blade aerodynamics. The signal has been filtered to include only the higher harmonic components, greater than 10 per rotor revolution.

\section{Wake Geometry}

The results already presented have shown that the VTM is able to accurately predict the high-frequency fluctuations in loading that are induced by BVIs on the rotor disk. Through a qualitative and quantitative analysis of the geometry of the wake, this section of the

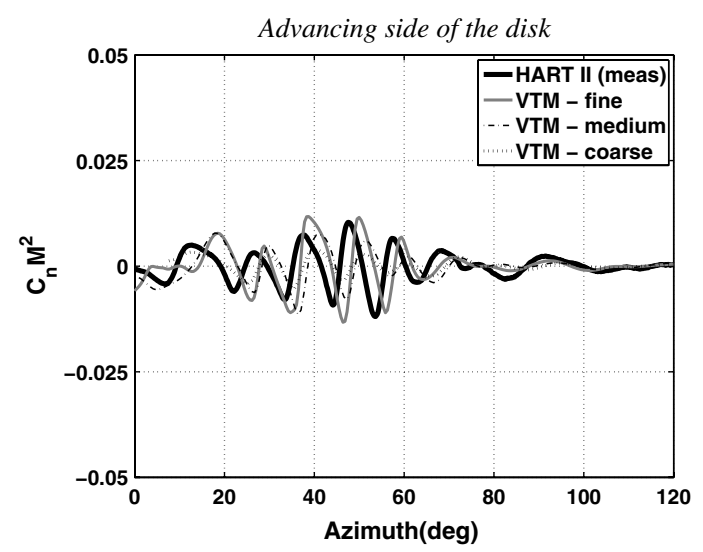

a)

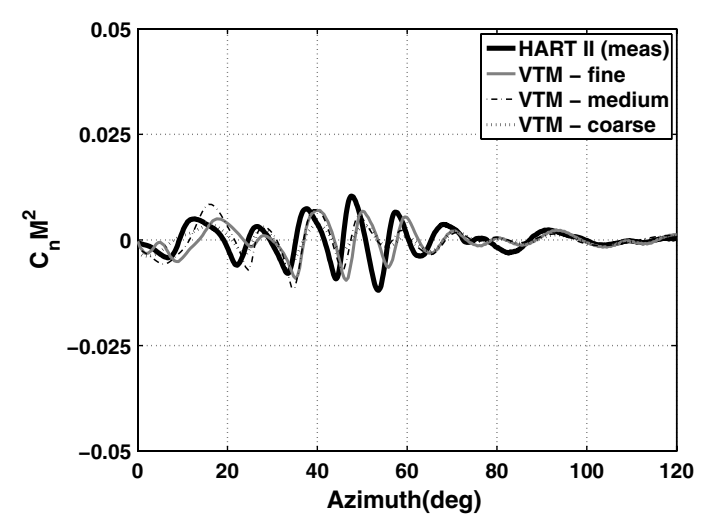

paper aims to confirm that this is due to the ability of the method to represent accurately the wake that is produced by the rotating blades. As the aerodynamic environment in which a rotor operates is extremely complex, the modeling of the evolution of the vortices within the wake, particularly in terms of their correct position and strength over time, is somewhat challenging.
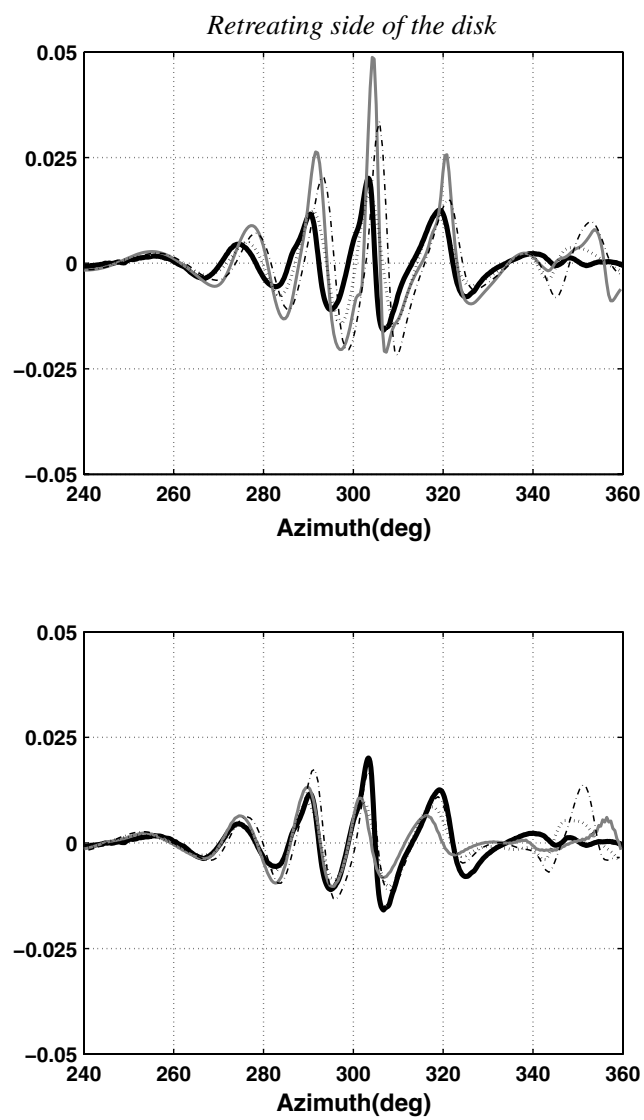

b)

Fig. 12 Comparison of blade loading $\left(C_{N} M^{2}\right)$ at $87 \%$ span against experimental data for the HART II BL case, as predicted using a) lifting-line and b) lifting-chord representations of the blade aerodynamics. The signal has been filtered to include only higher harmonic components greater than 10 per rotor revolution. 

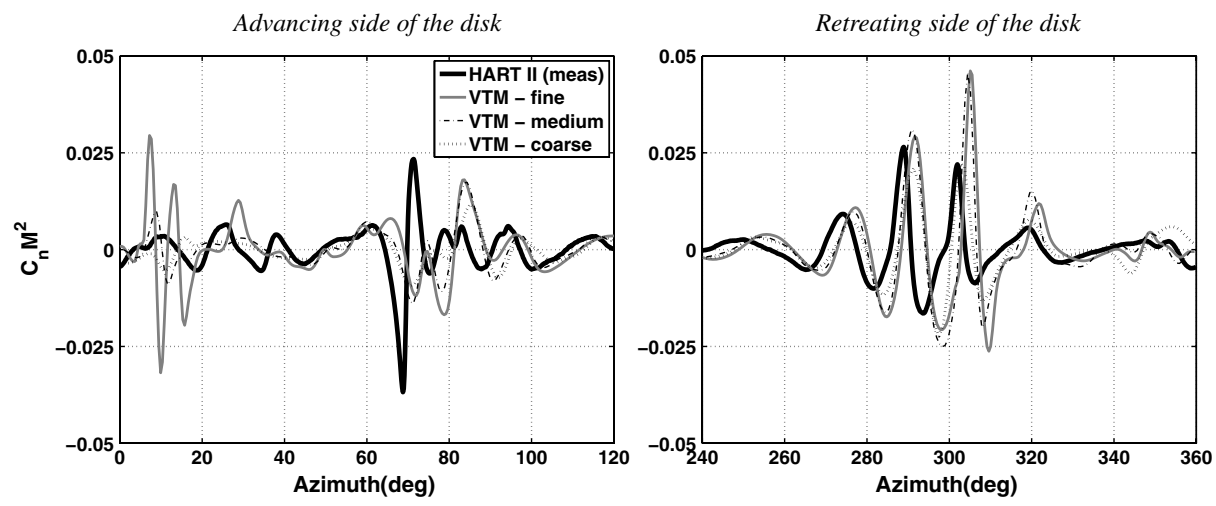

a)
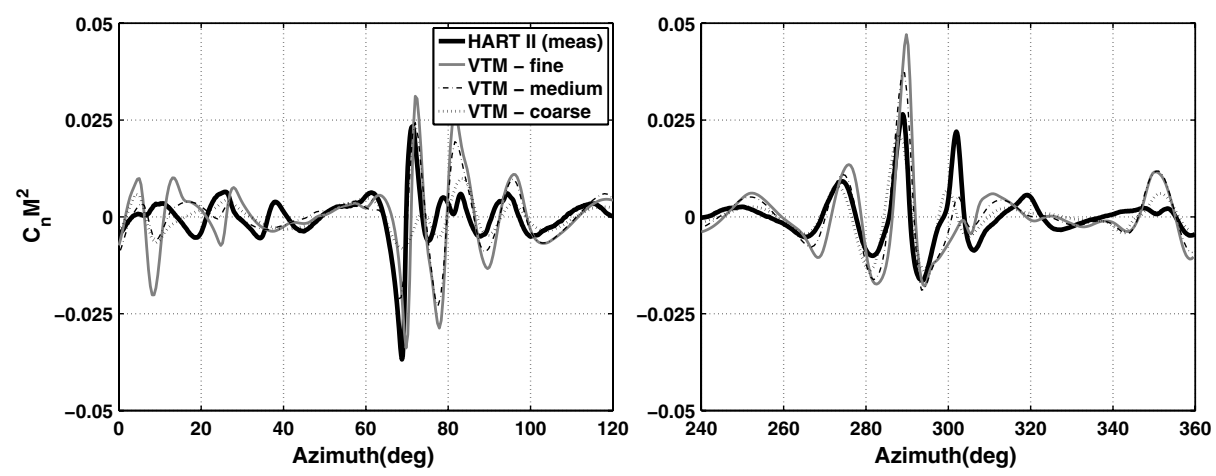

b)

Fig. 13 Comparison of blade loading $\left(C_{N} M^{2}\right)$ at $87 \%$ span against experimental data for the HART II MN case, as predicted using a) lifting-line and b) lifting-chord representations of the blade aerodynamics. The signal has been filtered to include only higher harmonic components greater than 10 per rotor revolution.
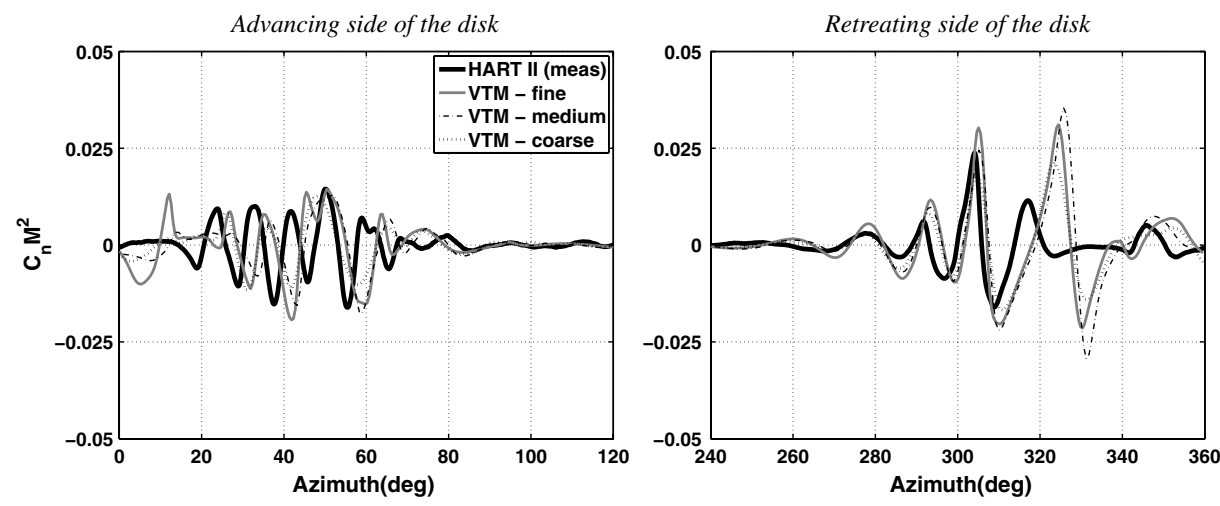

a)
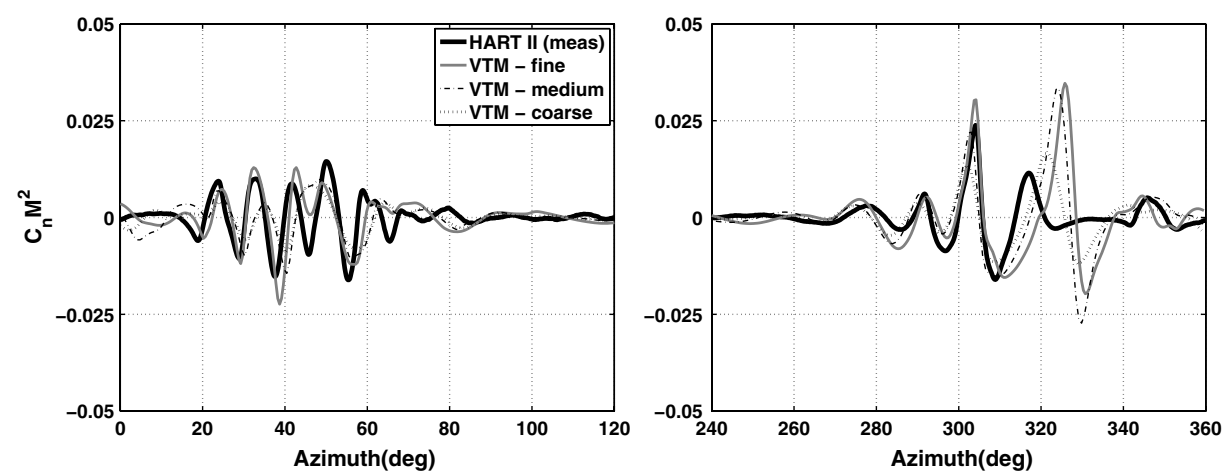

b)

Fig. 14 Comparison of blade loading $\left(C_{N} M^{2}\right)$ at $87 \%$ span against experimental data for the HART II MV case, as predicted using a) lifting-line and b) lifting-chord representations of the blade aerodynamics. The signal has been filtered to include only higher harmonic components greater than 10 per rotor revolution. 
The principal discrepancies in airload prediction described earlier are confined almost exclusively to the rear of the advancing side of the rotor. If errors in reproducing the deflections of the blades could be discounted, the observed discrepancies in airload prediction are thought to be primarily due to minor inaccuracies in modeling the development of the rotor wake, particularly the roll up of the vortex sheet immediately behind the blades to form the tip vortices on the advancing side of the rotor disk. The possible origins of these inaccuracies are explored by comparing the vortex geometry predicted by the VTM with that recorded during the HART II rotor test.

The geometry of the VTM-predicted wake of the HART II system at the medium computational resolution, as described in Table 2 , is visualized in Fig. 15 for each of the three test cases. A surface is plotted in the flow on which the vorticity has constant magnitude. This figure illustrates the characteristic behavior of the VTM, regardless of the model used for the aerodynamics of the rotor blade, in retaining the spatial compactness of the vortical structures that are present in the flow even after numerous rotor revolutions have elapsed. Indeed, it is this property of the model that makes the VTM particularly well suited to predicting loading perturbations on the scale of BVIs, as already shown. The modifications to the trajectories of the tip vortices, which result from the application of HHC, are clearly visible when comparing the resultant wake structure of the $\mathrm{MN}$ and MV cases with the BL case. In all three cases, the relatively strong root-vortex structure, as well as the broad vortex sheet that is generated behind the blades as they traverse the advancing side of the rotor, can be seen clearly. The image also reveals some detail of how this outboard sheet eventually rolls up to form a concentrated tip vortex at some distance behind the blades, particularly on the advancing side of the rotor. In the BL and MV cases, the BVI events on the retreating side of the disk are caused by near-parallel interactions between the rotor blade and the tip vortex. The modification to the tip-vortex trajectories that is introduced by the HHC in the MN case causes these vortices to reorientate so that they interact more obliquely with the rotating blades than in the BL case.

Figure 16 compares the wake structure that is predicted by the VTM at the medium resolution for the MN case when the lifting-
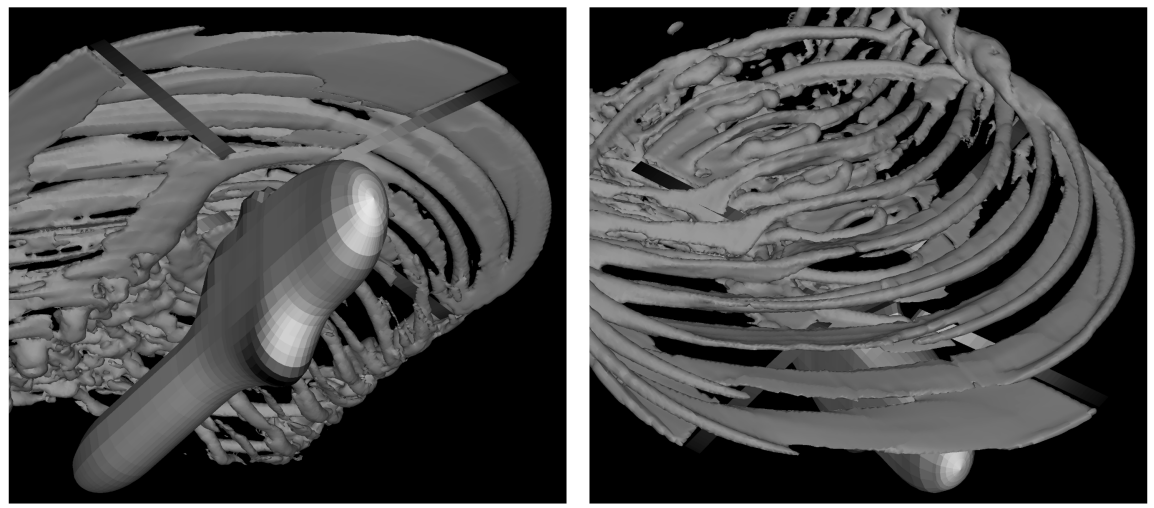

a) Baseline
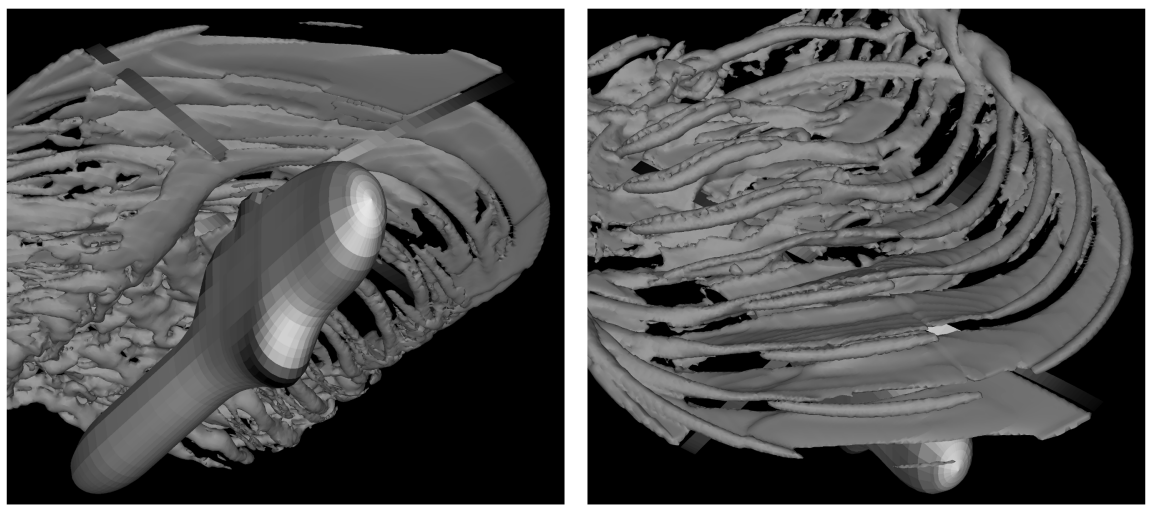

b) Minimum noise
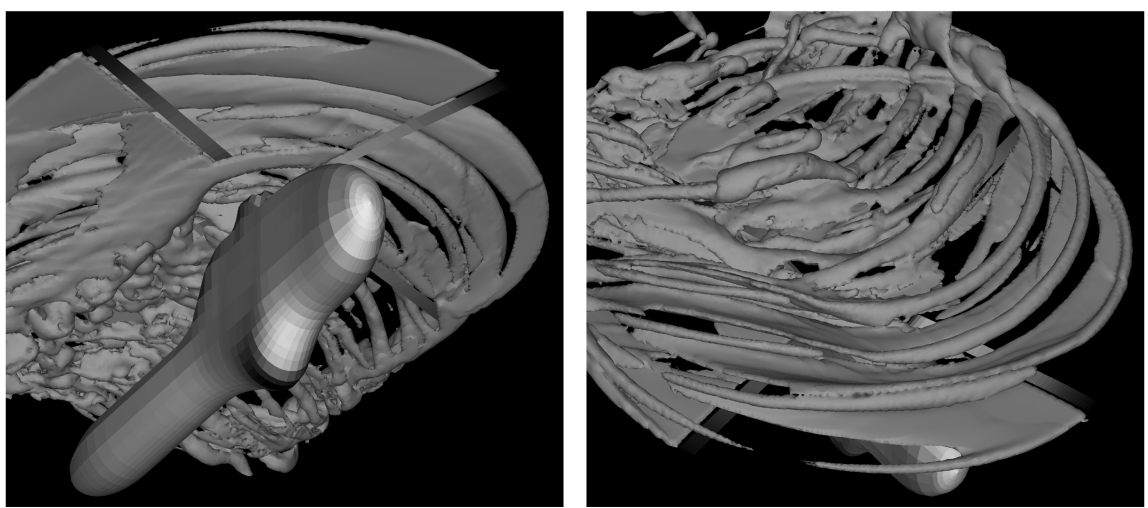

c) Minimum vibration

Fig. 15 Visualization of the VTM-predicted wake geometry from simulations of the HART II rotor at medium computational resolution. 


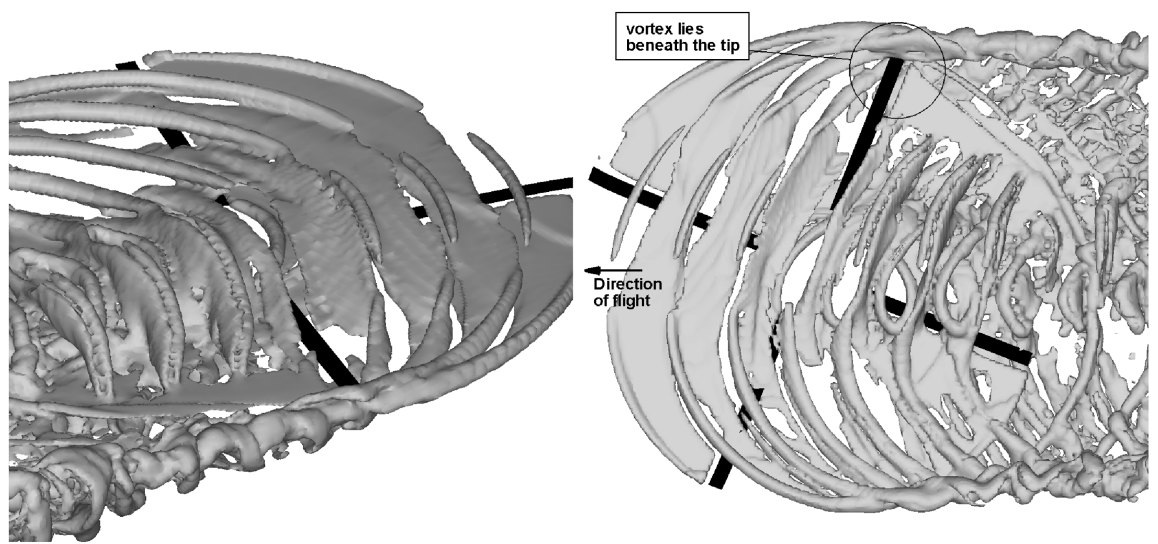

a) Lifting-line model

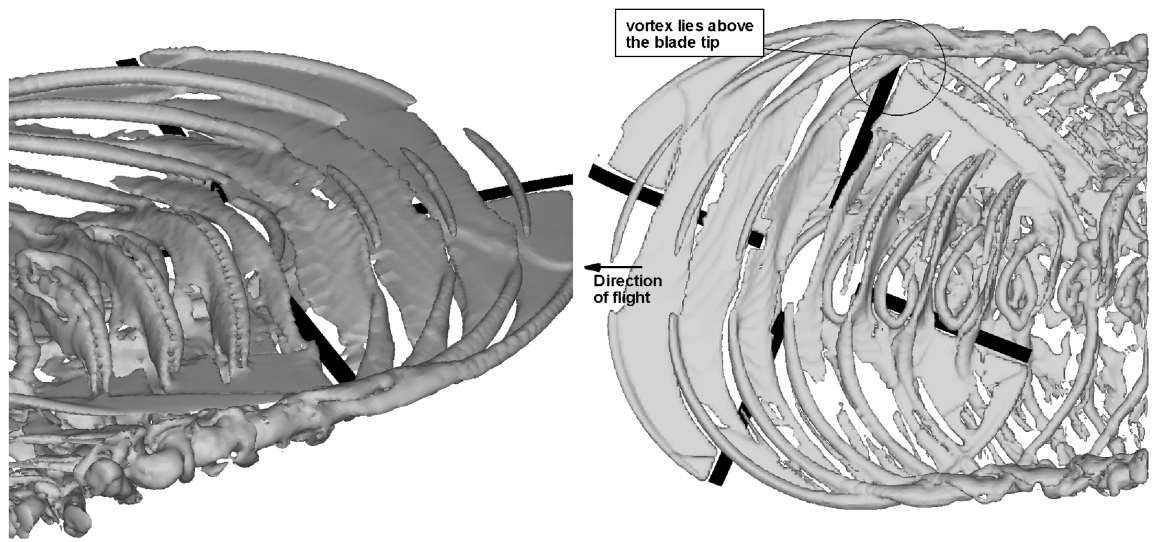

b) Lifting-chord model

Fig. 16 Geometry of the rotor wake with the reference blade at $70^{\circ}$ azimuth for the HART II MN case, as predicted using a) lifting-line and b) liftingchord representations of the blade aerodynamics.

chord representation of the aerodynamics of the blade is used with that predicted when the lifting-line model is used. This figure shows the sensitivity of the predicted wake structure to the model that is used to represent the blade aerodynamics. In the calculation in which the lifting-line model has been used, the vortex that is responsible for the most prominent BVI in the MN case passes beneath the blade, whereas when the lifting-chord model is used, the vortex passes above the blade. The VTM-predicted airloads are sensitive to the very subtle changes in the vorticity distribution that is sourced into the flow by the blades when different blade aerodynamic models are used; in this case, given the shallow angle between the vortex and the blade, it can easily be conceived how a small change in the predicted position of the vortex could have a large effect on the predictions of the associated BVI airload.

\section{Trajectory of Tip Vortices}

To demonstrate the ability of the method to resolve accurately the details of the wake and, in particular, the trajectories of the individual tip vortices as they are convected downstream and through the rotor disk, the computed wake structure is compared against measurements of the vortex core positions. A 3C-PIV technique [25] was used in the HART II test program to give instantaneous measurements of the velocity field contained within a series of highly

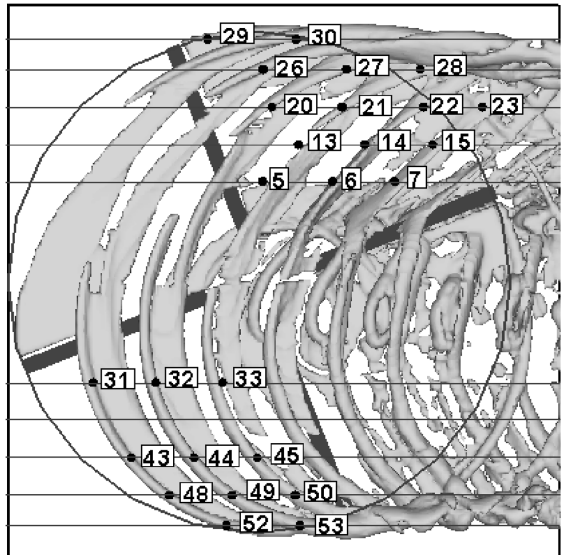

a) At $20^{\circ}$ azimuth

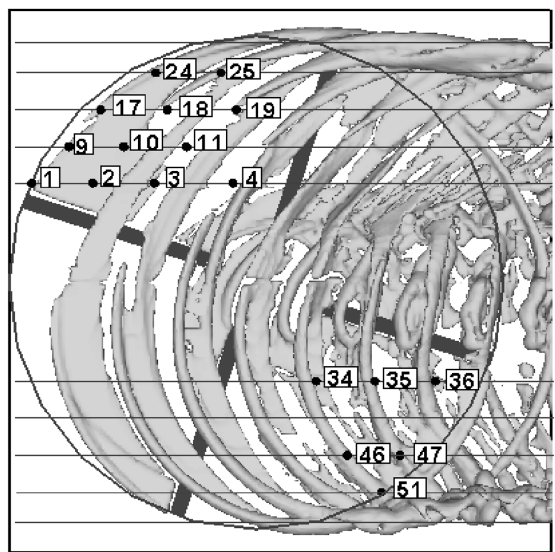

b) At $70^{\circ}$ azimuth

Fig. 17 Locations of PIV measurement planes superimposed on the wake structure predicted by the VTM for the HART II BL case. 

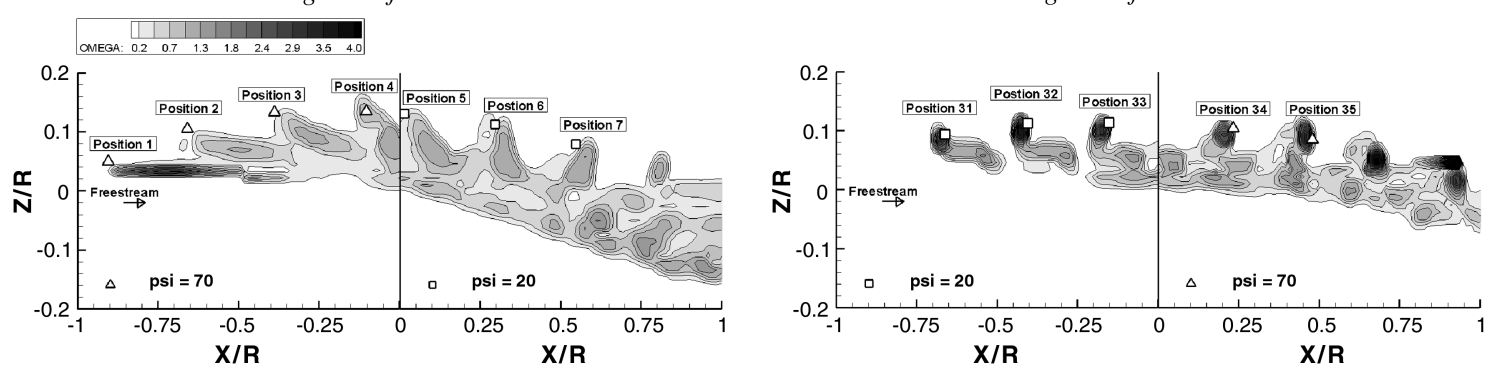

a) Lifting-line blade aerodynamic model
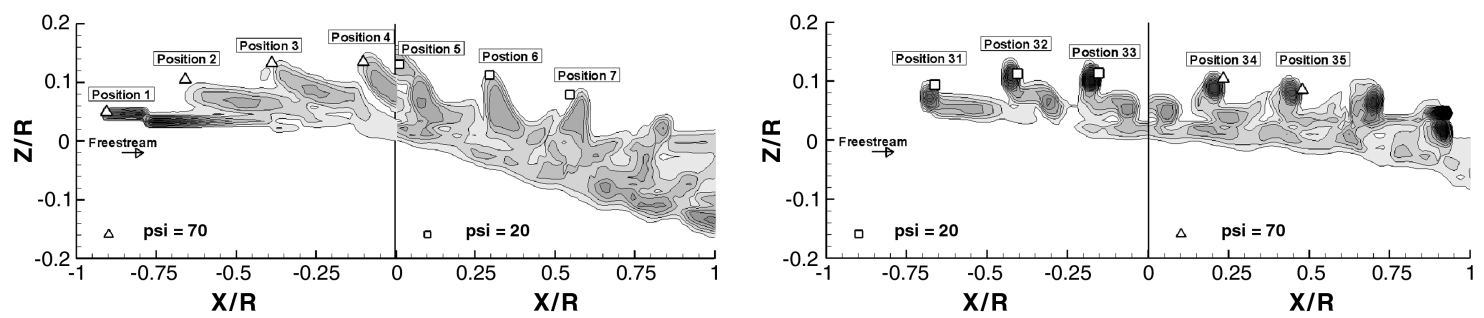

b) Lifting-chord blade aerodynamic model

Fig. 18 Computed wake structure (contours of vorticity magnitude) and vortex core positions (symbols) estimated from the experimental data compared on a longitudinal slice through the wake at $40 \%$ of the rotor radius (HART II BL case).

resolved observation areas near to the rotor. These observation areas were located along five longitudinal slices through the flow between $Y / R=0.4$ and $Y / R=0.97$, as indicated in Fig. 17. Figures 18 and 19 compare contour plots of vorticity magnitude, as predicted using each of the two models for the aerodynamics of the blades, with the experimental data that were measured on the two longitudinal slices located at 40 and $70 \%$ of the rotor radius. To prevent the rotor blades from obscuring the images, the PIV measurements at locations in the first and third quadrants were collected with the rotor at $20^{\circ}$ azimuth and, at locations in the second and fourth quadrants, with the rotor at $70^{\circ}$ azimuth. This difference accounts for the misalignment of the contours between the forward and aft sections of the rotor disk that is visible in the figures. Time lags in the data acquisition system gave an error of $3.5^{\circ}$ in the measured azimuthal position of the rotor, but this has been accounted for in the presentation of the data. The experimental measurements of the positions of the vortex cores are plotted

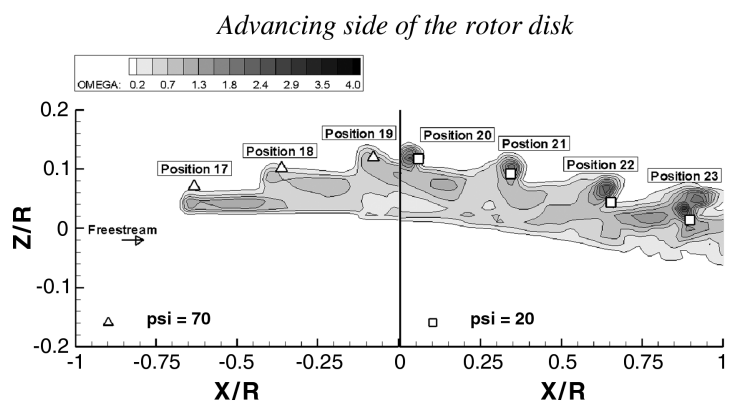

a) Lifting-line blade aerodynamic model

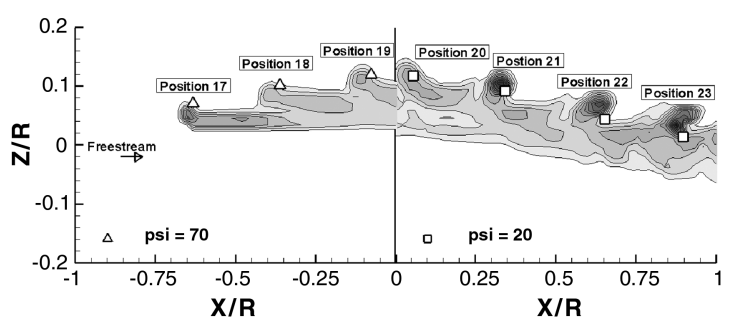

in Figs. 18 and 19 as symbols, and they are labeled with a number corresponding to the location of the PIV measurement plane (see Fig. 17). The positions of the vortex centers were identified from the simple average of approximately 100 PIV images of size 0.45 by $0.38 \mathrm{~m}(0.225 R$ by $0.19 R)$, as described in [25]. (In the right-handed hub coordinate system, $Z$ is positive upward and $X$ is positive aft. The rotor hub is located at the origin of the coordinate system.)

\section{A. Influence of Blade Aerodynamic Model}

Figures 18 and 19 illustrate the ability of the VTM to accurately capture the geometry of the rotor wake, irrespective of the method by which the blade loading is calculated, and hence by which the vorticity source into the flowfield is calculated. The locations of the maxima in the computed vorticity distribution in the wake show, in general, very good correlation with the experimentally measured

b) Lifting-chord blade aerodynamic model

\section{Retreating side of the rotor disk}
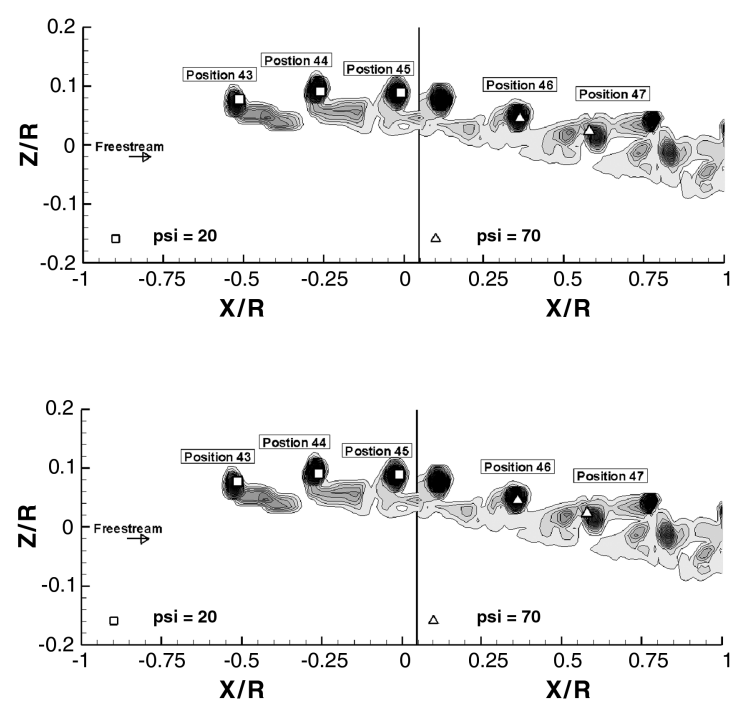

Fig. 19 Computed wake structure (contours of vorticity magnitude) and vortex core positions (symbols) estimated from the experimental data compared on a longitudinal slice through the wake at $70 \%$ of the rotor radius (HART II BL case). 
Advancing side of the rotor disk

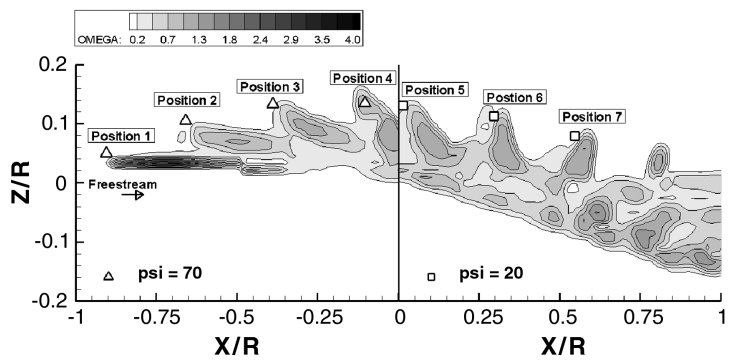

a) Medium computational resolution

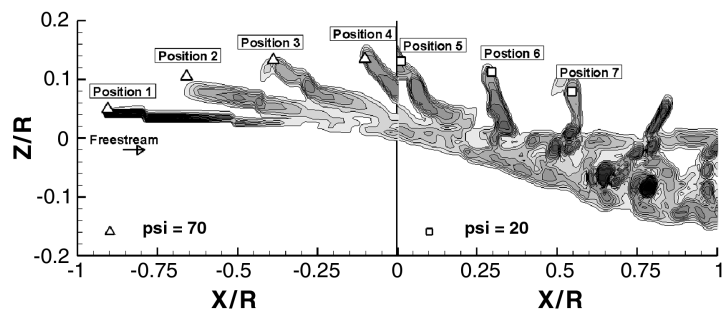

Retreating side of the rotor disk
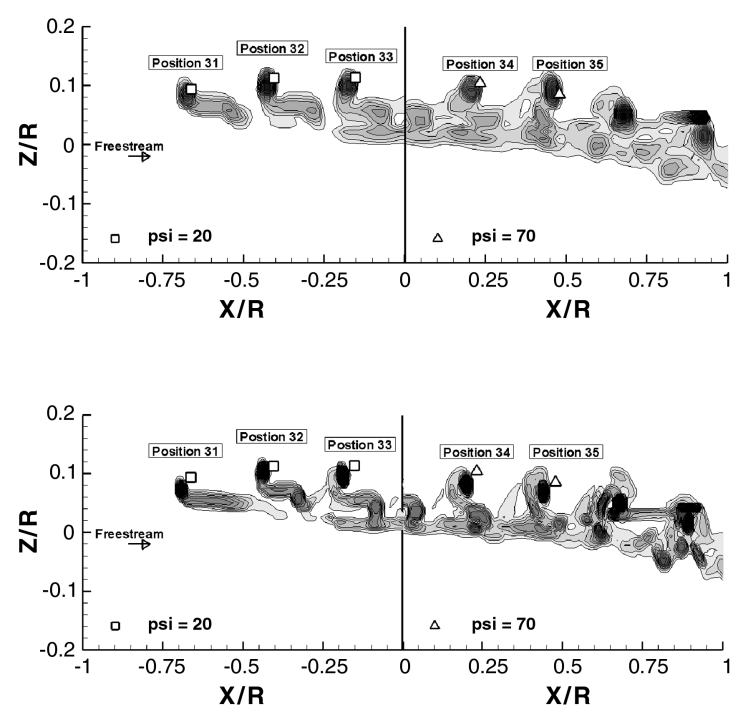

b) Fine computational resolution

Fig. 20 Computed structure of wake vorticity (contours) and measured vortex core positions (symbols) compared on longitudinal slices through the wake at $40 \%$ of the rotor radius, at medium and fine computational resolutions (HART II BL case).

vortex positions. This is true for the slices close to the rotor hub and for those which lie closer to the tips of the blades.

On the forward half of the rotor disk, the prediction of the vortex positions is better on the retreating side than on the advancing side of the rotor, which is consistent with the observed accuracy of the blade airload predictions. Such a trend is less obvious toward the rear of the rotor disk. Indeed, the positions of the vorticity maxima, as predicted by the VTM using both lifting-chord and lifting-line blade aerodynamic models, are all well within one chord length $(c / R=$ 0.0605 ) of the experimentally measured positions of the vortex cores.

The most obvious difference between the two different models for the loading on the blades is how they represent the structural features of the tip vortex when the vortex is relatively young: for example, when resolved on the observation planes at position 1 in Fig. 18 and

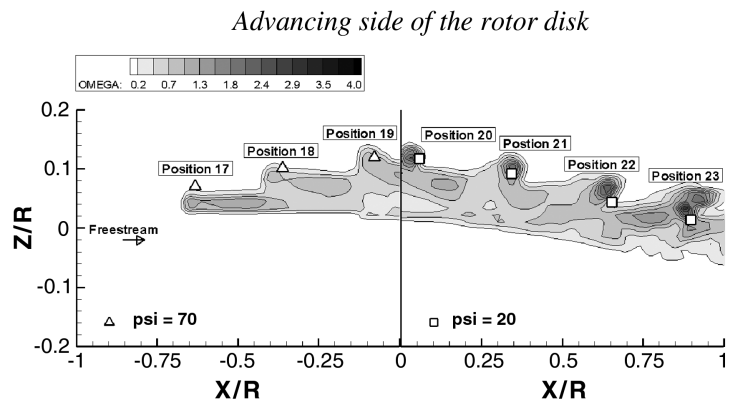

a) Medium computational resolution

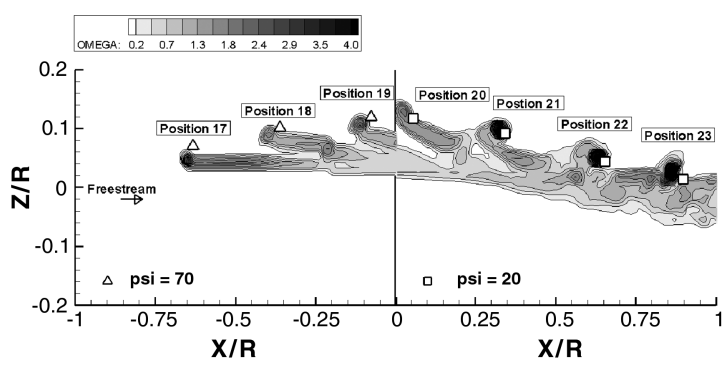

position 17 in Fig. 19. In both cases, the lifting-chord model more accurately predicts the position of the young tip vortex by capturing, to some extent, the slight curvature to the inboard sheet of vorticity as it rolls up just downstream of the advancing blade. With the grid resolutions that are presently achievable, these features are at the limit of what is currently possible to resolve.

It was postulated earlier that problems in resolving both the lowfrequency and the BVI-induced loading on the advancing side of the rotor could be due to misrepresentation of the root-vortex system that is generated by the rotor (perhaps as a result of the omission of any representation of the blade attachments or rotor hub in the simulations), and hence its effect in distorting the more outboard sections of the wake. This interpretation seems unlikely, however, in the light of the data presented in Fig. 18 for the wake structure at $40 \%$ of the

b) Fine computational resolution
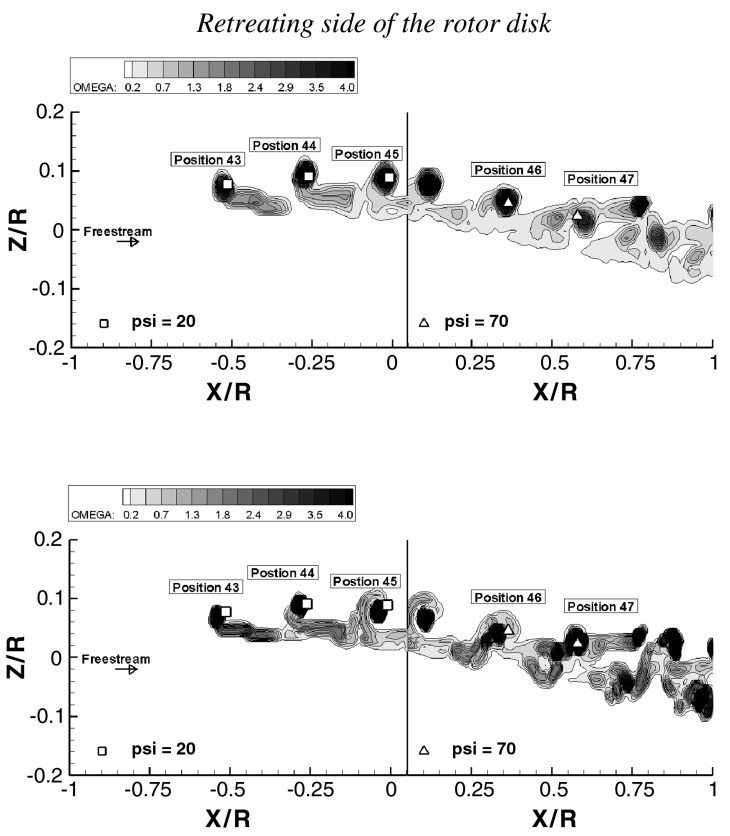

Fig. 21 Computed structure of wake vorticity (contours) and measured vortex core positions (symbols) compared on longitudinal slices through the wake at $70 \%$ of the rotor radius, at medium and fine computational resolutions (HART II BL case). 

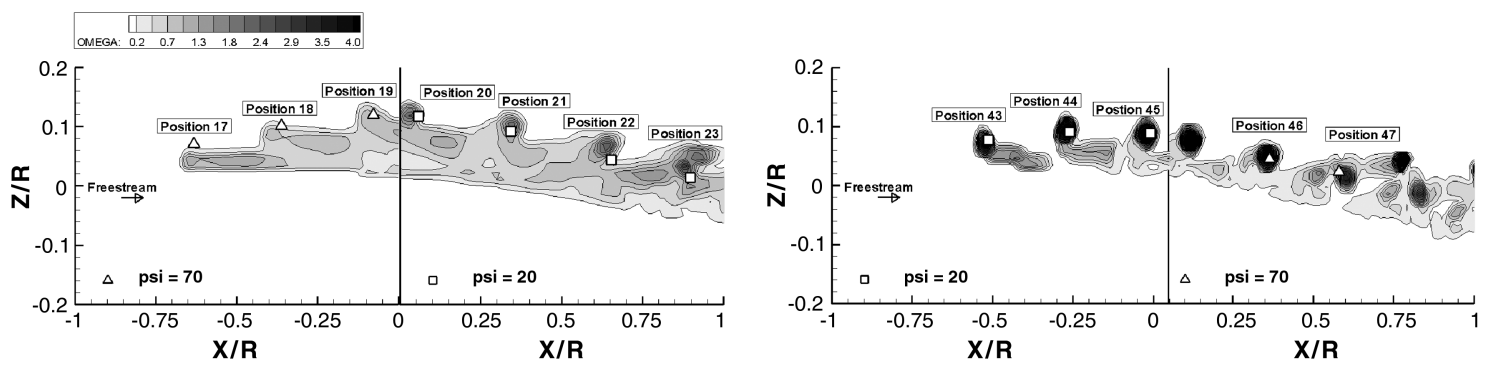

a) With a representation of the drive housing
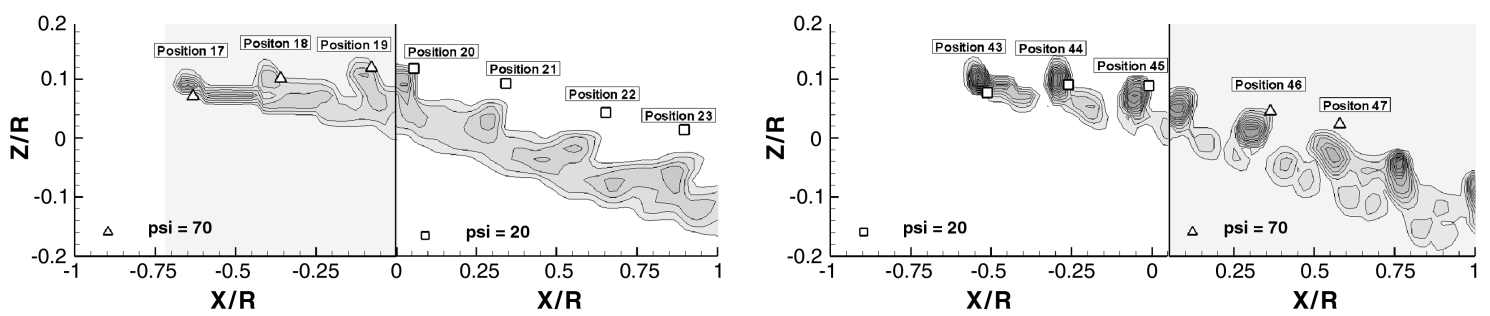

b) Isolated rotor

Fig. 22 Computed structure of wake vorticity (contours) and measured vortex core positions (symbols) compared on longitudinal slices through the wake at $70 \%$ of the rotor radius, with and without a representation of the drive enclosure (HART II BL case).

rotor span, in which the predicted vortex structure shows very good agreement with experimental data, even relatively close to the root cutout of the rotor.

\section{B. Effect of Grid Resolution}

A comparison of the results for medium and fine resolutions (as defined in Table 2) of the computational domain, shown in Figs. 20 and 21 , reveals that an increase in the spatial resolution of the flowfield results in a markedly improved definition of the various vortical structures within the wake, but it does not significantly alter the predicted positions of the vortex cores within the flow. The exception is on the advancing side of the rotor, particularly at $Y / R=0.4$, where a refinement of the computational mesh reduces the error in the prediction of the positions of the vortex cores from about one-third of the blade chord to within the resolution of the plotted data. On the other hand, cross-referencing the predictions of blade loading with the data presented here does suggest a consistency between the small improvement in vortex position that results from an increase in the resolution of the wake and a slight improvement in the phasing of the BVI-induced loading on the advancing side of the rotor disk. The concentration of the vorticity that is associated with each tip vortex, as the resolution of the flow domain is increased, lends some insight into the causes of the overpredictions in amplitude of the BVI-induced loading peaks, particularly when the lifting-line model for the blade aerodynamics is used.

\section{Effect of Including a Fuselage}

Figure 22 compares the vortex positions at $70 \%$ along the blade for the HART II BL case when an isolated rotor is modeled with those where a representation of the drive housing has been included in the
Advancing side of the rotor disk

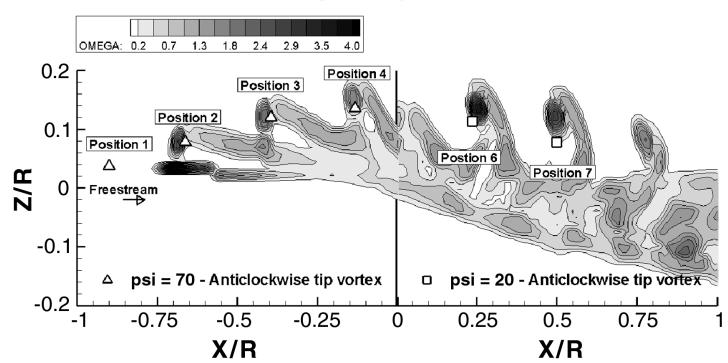

a) Lifting-line blade aerodynamic model

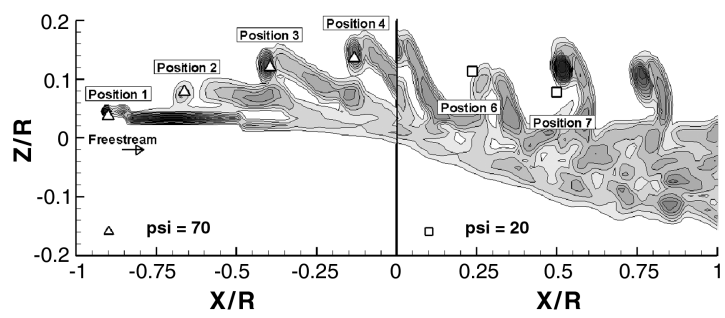

Retreating side of the rotor disk
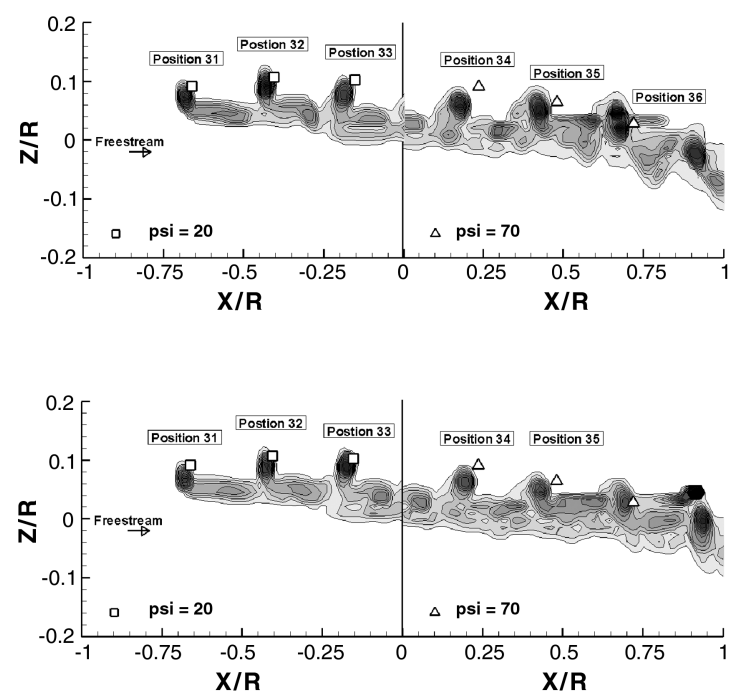

b) Lifting-chord blade aerodynamic model

Fig. 23 Computed wake structure (contours of vorticity magnitude) and vortex core positions (symbols) estimated from the experimental data compared on a longitudinal slice through the wake at $40 \%$ of the rotor radius (HART II MN case). 
Advancing side of the rotor disk

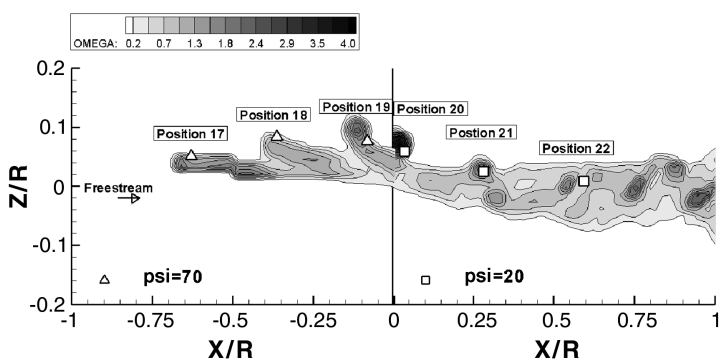

a) Lifting-line blade aerodynamic model

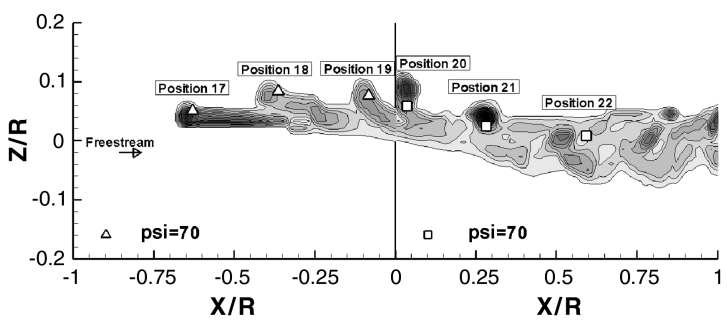

Retreating side of the rotor disk
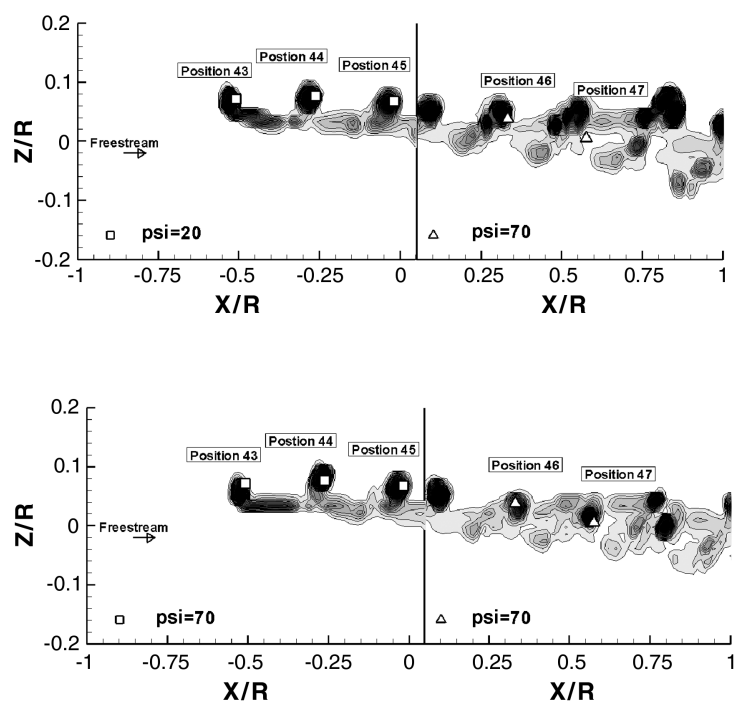

b) Lifting-chord blade aerodynamic model

Fig. 24 Computed wake structure (contours of vorticity magnitude) and vortex core positions (symbols) estimated from the experimental data compared on a longitudinal slice through the wake at $70 \%$ of the rotor radius (HART II MN case).

computation. Representing the drive housing in the computations results in an upward deflection of the predicted wake geometry compared with that when the fuselage is ignored, and it considerably improves the match between the experimentally measured and predicted vortex positions.

\section{Minimum Noise and Minimum Vibration Cases}

Figures 23 and 24 show contour plots of vorticity magnitude, as predicted using each of the two models for the aerodynamics of the blades, against experimental estimates of the positions of the vortex cores on each of the longitudinal slices where measurements were gathered for the $\mathrm{MN}$ case. The computation included a representation of the drive housing, and (as in the BL case) the locations of predicted maximum vorticity in the flow very accurately match the experimentally measured positions of the vortex cores. On the retreating side of the rotor, the vortices at position 47 and further downstream are perhaps deflected a little too strongly by the presence of the drive housing, resulting in vortex cores that lie slightly higher than the positions of the cores that were estimated from the experimental data.

The wake structure of the MV case, depicted in Figs. 25 and 26, is especially interesting. The experimental data suggest the existence of a twinned tip-vortex structure on the advancing side of the rotor disk. The existence of this structure was attributed to the reversed loading at the tip of the advancing blade under the conditions of this particular test. This is supported by the computational predictions, which show the vorticity distribution on the slice through the wake at $Y / R=0.7$ to consist of a sequence of paired vortices: one rotating clockwise and the other anticlockwise. The positions of both the clockwise and anticlockwise vortices are in good agreement with the experimental data until position 21; further aft, the anticlockwise tip vortices lie slightly higher than is observed experimentally. In contrast, the

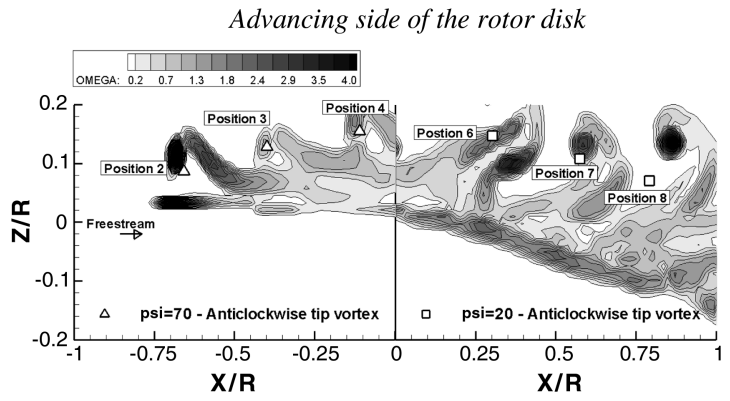

Retreating side of the rotor disk

a) Lifting-line blade aerodynamic model
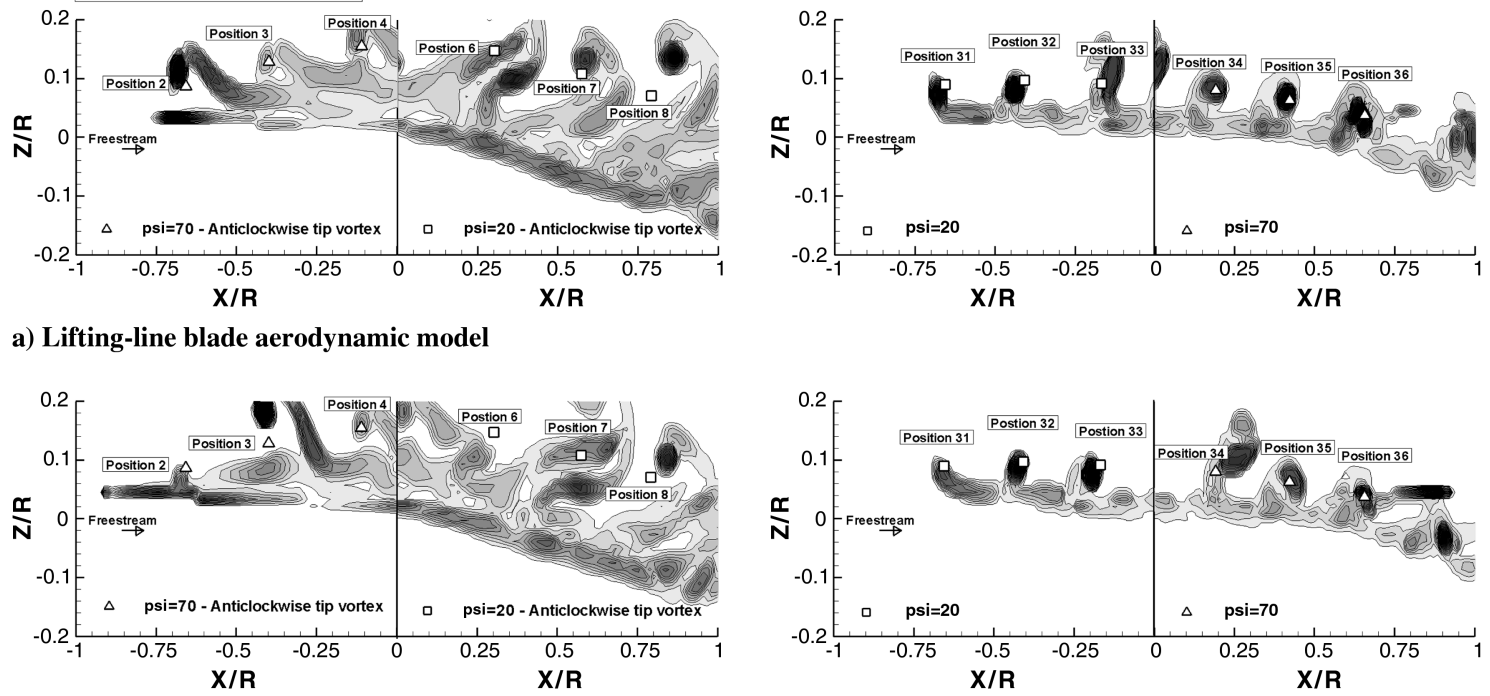

b) Lifting-chord blade aerodynamic model

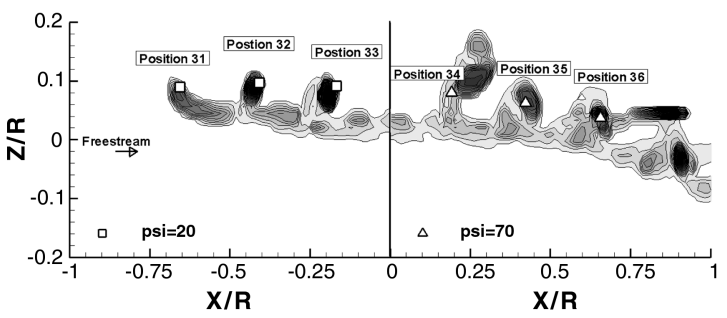

Fig. 25 Computed wake structure (contours of vorticity magnitude) and vortex core positions (symbols) estimated from the experimental data compared on a longitudinal slice through the wake at $40 \%$ of the rotor radius (HART II MV case). 
Advancing side of the rotor disk

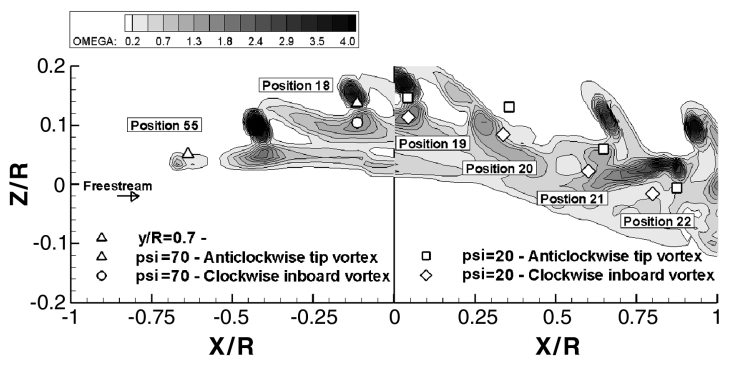

a) Lifting-line blade aerodynamic model

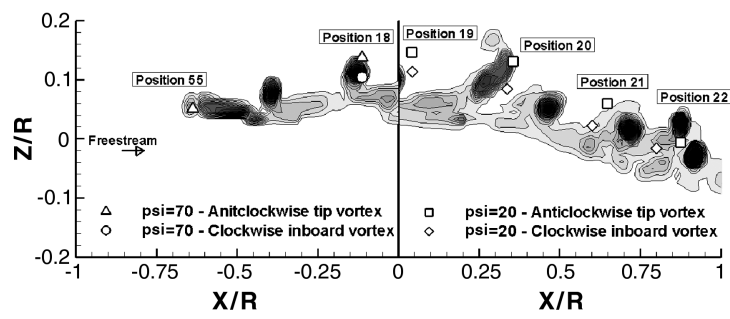

Retreating side of the rotor disk
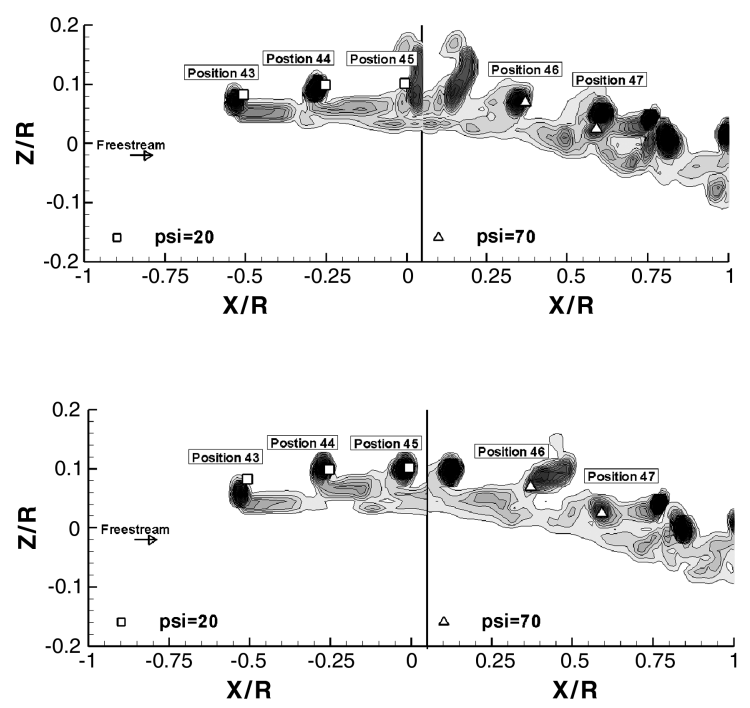

b) Lifting-chord blade aerodynamic model

Fig. 26 Computed wake structure (contours of vorticity magnitude) and vortex core positions (symbols) estimated from the experimental data compared on a longitudinal slice through the wake at $70 \%$ of the rotor radius (HART II MV case).

vortex positions at equivalent locations on the retreating side of the rotor are extremely well predicted by the computational method.

A rather revealing consistency is thus observed between the regions of the rotor where the major discrepancies in the predicted BVI-induced airload are encountered and where the prediction of the wake structure is at its least satisfactory. The results presented here serve to emphasize the extremely demanding requirements on the accuracy of the model of the rotor wake and the structural dynamics of the blades if truly accurate prediction of the BVI-induced airloads on the rotor is to result.

\section{Conclusions}

The effect of the fidelity of the blade aerodynamic model on the prediction of the high-frequency airloads that are associated with BVIs has been assessed by coupling the VTM to two different models for the aerodynamics of the blade. The first model is an extension of the Weissinger-L lifting-line model, in which the strength of a bound vortex, placed at the quarter chord of each blade panel, is determined by imposing a zero throughflow boundary condition at a single point located at the three-quarter chord of the panel. This approach is compared with a second lifting-chord method that is based on classical unsteady thin aerofoil theory. In this approach, the aerodynamic environment of the blade is represented by a series of weighted integrals over the chord of each blade panel.

In contrast to the lifting-line model, the lifting-chord model has been shown to accurately distinguish between those effects on the blade airloads that are due to a sudden change in angle of attack and those that are due to penetration of a vertical gust. The lifting-chord approach also more accurately represents the unsteady lift, in terms of both phase and amplitude, that is generated by an aerofoil in response to an encounter with an isolated, freely convecting vortex.

Predictions of blade airloads and wake structure, obtained using the VTM in conjunction with each of the blade aerodynamic models, have been compared against data gathered during the HART II experimental program. The rotor was operated in a descending flight condition in which the loading on its blades contained significant high-frequency content due to the presence of BVIs. These data have been used in the present paper to analyze the ability of each of the blade aerodynamic models to capture the detailed high-frequency BVI-induced loading on the rotor. The dynamics of the blades were prescribed to follow the experimentally measured blade motion, thus allowing any effects on the quality of the simulation that are due to structural deformations of the rotor blades to be separated from those that are induced by the aerodynamics of the system.

The quality of the prediction of the low-frequency component of the blade loading is negligibly influenced by the model that is used to represent the aerodynamics of the rotor blades. It is likely that the main discrepancies in the prediction of this component of the airload in the cases modeled here are simply due to a misrepresentation of the blade dynamics and are thus unrelated to the aerodynamic modeling of the system.

Where the lifting-line model was used to represent the aerodynamics of the blades, the predicted high-frequency BVI-induced component of the loading is found to be extremely sensitive to the cell size that is used in the computations. Although the phase and impulse width is only marginally influenced, the predicted amplitude of the BVI-induced features in the loading on the blades increases significantly as the cell size that is used to resolve the wake is reduced. Indeed, as the computational resolution is increased, the calculation significantly overpredicts the amplitude of the BVIinduced features in the loading, particularly on the retreating side of the rotor. These observations confirm that deficiencies in prediction of the BVI-induced airloads that were observed in earlier work were not simply due to underresolution of the flowfield, but they were also due to a misrepresentation of the aerodynamic response of the blade when subjected to the very localized perturbations in its aerodynamic environment that are characteristic of helicopter BVIs.

A marked improvement in the accuracy of the predicted high-frequency airloads of the HART II rotor is obtained when a lifting-chord model for the blade aerodynamics is used instead of the lifting-line-type approach. Errors in the amplitude and phase of the BVI-loading peaks are reduced, and the quality of the prediction is affected to a lesser extent by the computational resolution. In particular, the overprediction of the amplitude of the BVI events, which occurs on the retreating side of the disk as the resolution of the computation is increased when using the lifting-line model, is avoided. The insensitivity of the lifting-chord model to the resolution of the computation can be explained in terms of its reduced sensitivity to the localized small-scale features of the flowfield and the dependence of its predictions, rather than on the integral, invariant properties of the flowfield. In the context of helicopter BVI, the primary advantage of this approach would thus appear to be the possibility that it offers of true numerical convergence of predictions as the resolution of the computational grid is increased.

VTM predictions of the geometry of the wake are shown to match very closely with the experimental data, no matter which method is 
used to represent the aerodynamics of the rotor blades. The predicted positions of the vortex cores on two longitudinal slices through the flow, one situated close to the rotor hub and one further outboard along the blade span, agree with the measured data to within a fraction of the blade chord. The slight curvature of the sheet of vorticity behind the trailing edge of the blade is captured more accurately by the lifting-chord approach than by the lifting-line approach. Given the need for simpler models than CFD for use in design, this paper reveals how carefully the wake structure and the aerodynamic response of the rotor blades must be matched if accurate predictions of the high-frequency airloads that are principally responsible for the objectionable characteristics of helicopter rotor noise are to be achieved.

\section{References}

[1] Boyd, D. D., "HART II Acoustic Predictions Using a Coupled CFD/ CSD Method," Proceedings of the American Helicopter Society 65th Annual Forum [CD-ROM], Grapevine, TX, American Helicopter Soc., Washington, D.C., 2009.

[2] Lim, J. W., and Strawn, R. C., "Prediction of HART II Rotor BVI Loading and Wake System Using CFD/CSD Loose Coupling," Proceedings of the 45th AIAA Aerospace Sciences Meeting and Exhibit, AIAA Paper 2007-1281, Reno, NV, 2007.

[3] van der Wall, B. G., Junker, B., Burley, C., Brooks, T., Yu, Y. H., Tung, C., Raffel, M., Richard, H., Wagner, W., Mercker, E., Pengel, K., Holthusen, H., Beaumier, P., and Delrieux, Y., "The HART II Test in the LLF of the DNW: A Major Step Towards Rotor Wake Understanding," Proceedings of the 28th European Rotorcraft Forum [CD-ROM], Bristol, England, Royal Aeronautical Soc., London, 2002.

[4] van der Wall, B. G., Burley, C., Yu, Y. H., Richard, H., Pengel, K., and Beaumier, P., "The HART II Test: Measurement of Helicopter Rotor Wakes," Aerospace Science and Technology, Vol. 8, No. 4, 2004, pp. $273-284$. doi:10.1016/j.ast.2004.01.001

[5] Lim, J. W., Tung, C., Yu, Y. H., Burley, C., Brooks, T., Boyd, D., van der Wall, B. G., Schneider, O., Richard, H., Raffel, M., Beaumier, P., Delrieux, Y., Pengel, K., and Mercker, E., "HART II: Prediction of Blade-Vortex Interaction Loading," Proceedings of the 29th European Rotorcraft Forum [CD-ROM], Friedrichshafen, Germany, Deutsche Gesellschaft fur Luft- und Raumfahrt, Cologne, Germany, 2003.

[6] Yu, Y. H., Tung, C., van der Wall, B. G., Pausder, H., Burley, C., Brooks, T., Beaumier, P., Delrieux, Y., Mercker, E., and Pengel, K., "The HARTII Test: Rotor Wakes and Aeroacoustics with Higher-Harmonic Pitch Control (HHC) Inputs: The Joint German/French/Dutch/U.S. Project," Proceedings of the American Helicopter Society 58th Annual Forum [CD-ROM], Montreal, American Helicopter Soc., Washington, D.C., 2002.

[7] Hopkins, A. S., and Ormiston, R. A., "An Examination of Selected Problems in Rotor Blade Structural Mechanics and Dynamics," Proceedings of the American Helicopter Society 59th Annual Forum [CD-ROM], Phoenix, AZ, American Helicopter Soc., Washington, D.C., 2003.

[8] Johnson, W., "Rotorcraft Aerodynamics Models for a Comprehensive Analysis," Proceedings of the American Helicopter Society 54th Annual Forum [CD-ROM], Washington, D.C., American Helicopter Soc., Washington, D.C., 1998.

[9] Benoît, B., Dequin, A.-M., Kampa, K., Grunhagen, W., Basset, P.-M., and Gimonet, B., "HOST, a General Helicopter Simulation Tool for Germany and France," Proceedings of the American Helicopter Society 56th Annual Forum, Virginia Beach, VA, 2002.

[10] Brown, R. E., and Line, A. J., "Efficient High-Resolution Wake Modelling Using the Vorticity Transport Model," AIAA Journal,
Vol. 43, No. 7, 2005, pp. 1434-1443. doi: $10.2514 / 1.13679$

[11] Brown, R. E., "Rotor Wake Modeling for Flight Dynamic Simulation of Helicopters," AIAA Journal, Vol. 38, No. 1, 2000, pp. 57-63. doi: $10.2514 / 2.922$

[12] Whitehouse, G., Boschitsch, A., Quackenbush, T., Wachspress, D., and Brown, R. E., "Novel Eulerian Vorticity Transport Wake Module for Rotorcraft Flow Analysis," Proceedings of the American Helicopter Society 63rd Annual Forum [CD-ROM], Virginia Beach, VA, American Helicopter Soc., Washington, D.C., 2007.

[13] Peters, D. A., Hsieh, M.-C., and Torrero, A., "A State-Space Airloads Theory for Flexible Airfoils," Journal of the American Helicopter Society, Vol. 52, No. 4, 2007, pp. 329-342. doi:10.4050/JAHS.52.329

[14] Toro, E. F., "A Weighted Average Flux Method for Hyperbolic Conservation Laws," Proceedings of the Royal Society of London, Series A: Mathematical and Physical Sciences, Vol. 423, No. 1865, 1989, pp. 401-418. doi:10.1098/rspa.1989.0062

[15] Kenyon, A. R., and Brown, R. E., "Wake Dynamics and RotorFuselage Aerodynamic Interactions," Journal of the American Helicopter Society, Vol. 54, No. 1, 2009, Paper 012003. doi:10.4050/JAHS.54.012003

[16] Schneider, O., "Analysis of SPR Measurements from HART II," Aerospace Science and Technology, Vol. 9, No. 5, 2005, pp. 409420.

doi:10.1016/j.ast.2005.01.013

[17] Schneider, O., van der Wall, B. G., and Pengel, K., "HART II Blade Motion Measured by Stereo Pattern Recognition (SPR)," Proceedings of the American Helicopter Society 59th Annual Forum [CD-ROM], Phoenix, AZ, American Helicopter Soc., Washington, D.C., 2003.

[18] Pengel, K., Müller, R., and van der Wall, B. G., "Stereo Pattern Recognition: The Technique for Reliable Rotor Blade Deformation and Twist Measurement," Proceedings of the AHS International Meeting on Advanced Rotorcraft Technology and Life Saving Activities (Heli Japan) [CD-ROM], Tochigi, Utsunomiya, Japan, American Helicopter Soc., Washington, D.C., 2002.

[19] Kelly, M. E., Duraisamy, K., and Brown, R. E., "Predicting Blade Vortex Interaction, Airloads and Acoustics Using the Vorticity Transport Model," Proceedings of the 9th American Helicopter Society Aeromechanics Specialists' Meeting [CD-ROM], San Francisco, CA, American Helicopter Soc., Washington, D.C., 2008.

[20] Leishman, J. G., Principles of Helicopter Aerodynamics (Cambridge Aerospace Series), 2nd ed., Cambridge Univ. Press, New York, 2006.

[21] Wagner, H., "Über die Entstehung des Dynamischen Auftriebes von Tragflügeln," Zeitschrift für Angewandte Mathematik und Mechanik, Vol. 5, No. 1, 1925, pp. 17-35. doi:10.1002/zamm.19250050103

[22] Küssner, H. G., "Zusammenfassender Bericht über den Instationären Auftrieb von Flügeln," Luftfahrt-Forschungsberichte des Bundesministers für Verkehr, Vol. 13, No. 12, 1936, pp. 410-424.

[23] Duraisamy, K., and Brown, R. E., "Aerodynamic Response of a Hovering Rotor to Ramp Changes in Pitch Input," Proceedings of the American Helicopter Society 64th Annual Forum [CD-ROM], Montreal, American Helicopter Soc., Washington, D.C., 2008.

[24] Thom, A., and Duraisamy, K., "High Resolution Computation of the Aerodynamics and Acoustics of Blade Vortex Interaction," Proceedings of the American Helicopter Society 65th Annual Forum [CD-ROM], Grapevine, TX, American Helicopter Soc., Washington, D.C., 2009.

[25] van der Wall, B. G., and Richard, H., "Analysis Methodology for 3C PIV Data of Rotary Wing Vortices," Experiments in Fluids, Vol. 40, No. 5, 2006, pp. 798-812. doi:10.1007/s00348-006-0117-x 